\title{
A strong test of the dual-mode hypothesis
}

\author{
ERIN M. INGVALSON and MICHAEL J. WENGER \\ University of Notre Dame, Notre Dame, Indiana
}

\begin{abstract}
One account of facial cognition, the dual-mode hypothesis, maintains that there are two sources of information in a human face, featural and configural, and that these sources are processed simultaneously and independently of one another. According to the hypothesis, the processing and identification of upright faces relies primarily on configural information, and this information is disrupted to such an extent upon inversion as to result in a reliance on featural information for identifying inverted faces (e.g., Searcy \& Bartlett, 1996). When considered in terms of the general characteristics of human information processing, the foundational assumptions of the dual-mode hypothesis are as follows: Facial information processing is accomplished by a parallel self-terminating architecture with unlimited capacity to supercapacity and a preservation of independence between the rates of processing of featural and configural information. Although a number of studies have provided evidence consistent with the dual-mode hypothesis, until now there have been no direct tests of the foundational assumptions of the hypothesis. The present study provides that direct test, providing strong support for three of the assumptions (parallel self-terminating processing with unlimited capacity to supercapacity) while contradicting a fourth (independence in rates).
\end{abstract}

Our everyday experience with human faces suggests the importance of and the ability to deal with both the anatomical features of a face and their arrangement or configuration. For example, a person of dubious character might be described as having dark, close-set eyes. Yet there have been, and continue to be, arguments in the literature on facial perception and memory regarding the extent to which one or the other of these types of information may be critical to performance (e.g., Cabeza \& Kato, 2000; Hosie, Ellis, \& Haig, 1988; Macho \& Leder, 1998; Parks, Coss, \& Coss, 1985; Rhodes, 1988; Suzuki \& Cavanagh, 1995; Tanaka \& Sengco, 1997). One approach that has been taken in an attempt to consider the simultaneous roles of featural and configural information is the dual-mode hypothesis (e.g., Bartlett \& Searcy, 1993; Searcy \& Bartlett, 1996). ${ }^{1}$ According to this hypothesis, there exist two modes for processing faces, where "[o]ne mode is specialized for the encoding [of] spatial-relational information, whereas the other is specialized for the encoding of components" (Searcy \& Bartlett, 1996, p. 905). Both modes are viable in processing upright faces, but the featural mode prevails in the processing of inverted faces. According to the hypothesis, spatial relational informa-

Portions of this work were presented at the 35th Annual Meeting of the Society for Mathematical Psychology, Miami University of Ohio, July 2002. Sincere thanks go to Colin Crossman for his assistance in the creation of the stimuli and to Angelina Copeland and three anonymous reviewers for their helpful comments on an earlier version of this article. Correspondence can be directed to either E. M. Ingvalson, Department of Psychology, Carnegie Mellon University, Pittsburgh, PA 15213 (e-mail: eingvals@andrew.cmu.edu) or M. J. Wenger, Department of Psychology, Pennsylvania State University, 620 Moore Bldg., University Park, PA 16802 (e-mail: mjw19@psu.edu). tion is most easily processed in an upright orientation, with inversion producing decrements in the ability to process this type of information, relative to the ability to process component feature information.

Descriptions of the dual-mode hypothesis follow the modal practice in the (psychological) face-processing literature by defining featural information in terms of the anatomical features of a face, such as the eyes and the mouth. Configural information refers to the spatial relations among the anatomical features and has also been referred to as holistic information (e.g., Bartlett \& Searcy, 1993; Garner, 1978), second order spatial relations (e.g., Diamond \& Carey, 1986), relational information (e.g., Leder \& Bruce, 1998), and spatial relational information (e.g., Searcy \& Bartlett, 1996). Searcy and Bartlett defined spatial relational information as the information that is altered when the features in a face are moved or changed in size; Leder and Bruce (2000) referred to this information as configural information, the convention adopted here. By these definitions, a manipulation of featural information consists of altering the appearance of the features by, for example, blackening out some of the teeth or changing eye color (e.g., Bartlett \& Searcy, 1993). Similarly, a configural alteration consists of any change to the spatial relations among the features and is frequently implemented by, for example, moving the mouth down or the eyes farther apart (e.g., Bartlett \& Searcy, 1993), changes that would be physically impossible in an actual face.

The dual-mode hypothesis, as it is currently framed, makes three critical or core assumptions regarding the processing of featural and configural information. These assumptions are as follows: (1) Featural and configural sources of information are simultaneously available when a face is viewed, independently of the orientation of the 
face ${ }^{2}(2)$ the featural and configural sources are processed in an independent manner; and (3) orientation of the image determines which of these two sources will dominate processing (Leder \& Bruce, 2000; Searcy \& Bartlett, 1996).

\section{Evidence Supporting the Dual-Mode Hypothesis}

One of the motivations for the dual-mode hypothesis was Thompson's (1980) Thatcher effect. This effect is observed by inverting the eyes and mouth in an upright face, resulting in a grotesque appearance that is minimized when the entire face is inverted. Three accounts of this effect have been given which included the disruption of facial expression (e.g., Valentine, 1991; Yin, 1969), the use of reference frames (e.g., Parks et al., 1985), and distinctions between configural and featural information and the effects of inversion on each (e.g., Carey \& Diamond, 1977; Sergent, 1984).

Bartlett and Searcy (1993) tested the dual-mode hypothesis against the disruption-of-expression hypothesis and the reference frame hypothesis. Over the course of three experiments, they determined that configural and featural information were both available and used in rating or categorizing upright pairs but that inversion disrupted configural information to such an extent that only featural information was used in rating and categorizing inverted pairs. Rhodes, Brake, and Atkinson (1993) found the same relationship between configural and featural information, using a recognition task along with a rating task. This set of results is consistent with the first of the three core assumptions of the dual-mode hypothesis.

A prominent alternative view-the holistic-encoding hypothesis (e.g., Farah, Wilson, Drain, \& Tanaka, 1998; Tanaka \& Farah, 1993; Tanaka \& Sengco, 1997)accounts for the Thatcher effect by proposing that faces are encoded and processed as gestalts. In this view, there is no fundamental distinction between featural and configural information. Upright faces tend to be encoded as unitary wholes, whereas inversion changes the encoding process, diminishing the probability that the image will be encoded in a holistic fashion. Macho and Leder (1998) contrasted this account with the dual-mode hypothesis. They focused on the extent to which observers can store an individual feature in memory, something that is possible according to the dual-mode account but that is far less likely according the holistic-encoding hypothesis. The critical conclusion from this work was that individual feature information is stored in memory without interacting with configural information. This conclusion is consistent with the first two core assumptions of the dual-mode hypothesis.

Searcy and Bartlett (1996) investigated the premise that inversion disrupts configural information to a greater extent than it disrupts featural information. Using configurally and featurally distorted faces, they repeated the rating and paired-comparison tasks used in earlier work (Bartlett $\&$ Searcy, 1993), with very similar results. Configurally altered and inverted faces received lower grotesqueness ratings and were more likely to be rated as similar to a normal inverted face or another configurally altered face. These two findings were not found for featurally altered faces, although there was a small but significant increase in latencies for inverted pairs, consistent with the Rhodes et al.'s (1993) conclusion of inversion effects for featural information. Searcy and Bartlett also recorded response latencies from the paired-comparison tasks; analyses of these data suggested that participants had much greater difficulty evaluating configurally altered inverted faces than their upright counterparts, as evidenced by a significant decrease in the ability to respond in the allotted time interval and an increase in latencies for the responses that did occur within the limits. When the configural alterations were made more dramatic (Experiment 3), participants were better able to evaluate an inverted spatially altered face than before, although their response latencies were still significantly lower than those for configurally altered upright faces. Taken together, this evidence supports the claim that inversion has a very negative and selective impact on the processing of configural information (Searcy \& Bartlett, 1996), consistent with the third of the core assumptions of the dual-mode hypothesis.

In an effort to determine whether observers use two sources of information in a face or simply use the face as a whole, Cabeza and Kato (2000) tested the dual-mode hypothesis (e.g., Searcy \& Bartlett, 1996) against the holistic-encoding hypothesis (e.g., Farah et al., 1998), using the prototype effect - that is, the finding that after studying a group of objects, people tend to identify a composite, or prototype, of the objects as old, even though it was not part of the studied group. They created a set of featural and configural prototypes from four faces in the studied group and then presented these prototypes, studied faces, nonstudied faces, and prototypes of nonstudied faces in the test portion. When the faces were presented upright, the participants were equally likely to call both studied and nonstudied prototypes old, leading them to conclude that featural and configural information are independently processed and equally available when faces are presented upright. However, when the faces were inverted, the featural prototypes continued to elicit old responses, whereas the configural prototypes elicited fewer old responses, relative to upright presentation. Thus, the evidence collected by Cabeza and Kato is consistent with all three of the assumptions of the dual-mode hypothesis.

Additional evidence consistent with Searcy and Bartlett's (1996) results and with the assumptions of the dual-mode hypothesis were provided by Leder and Bruce (2000). Data from a rating task suggested that featural and configural information are used in evaluating facial distinctiveness and that the processing of configural information is negatively affected by inversion. Data from a recognition task suggested that the recognition of upright faces was supported primarily by configural information. Featural information appeared to dominate when 
the images were inverted, due to the presumed disruption of the ability to use configural information. The presence of featural information helped to lessen the inversion effect on configural information somewhat, but not completely (Leder \& Bruce, 2000).

\section{The Dual-Mode Hypothesis as a Processing Hypothesis}

Although the evidence above is consistent with all three assumptions and provides support for the dualmode hypothesis, it is all indirect evidence. That is, none of the studies discussed above (1) involved a logical or formal analysis of the complete set of alternative of hypotheses and (2) used experimental procedures that could allow for strong-inference (e.g., Platt, 1964; Popper, 1935) tests of those hypotheses (i.e., falsification of all but one of the alternative hypotheses). Without this type of analysis and these types of tests, not only are alternative and possibly contradictory explanations viable, but also it is not clear what the set of alternative conceptions might be. The present study provides the first and (to our knowledge) only strong-inference test of the dualmode hypothesis and does so by starting with a logical analysis of the hypothesis in terms of fundamental characteristics of information processing (following Townsend, 1974; Townsend \& Ashby, 1983; Townsend \& Nozawa, 1995).

Analyzing and formalizing the dual-mode hypothesis in terms of the fundamental characteristics of information processing allows us to take advantage of almost 3 decades worth of important theoretical and methodological advances (e.g., Schweickert, 1978, 1983; Schweickert, Giorgini, \& Dzhafarov, 2000; Schweickert \& Townsend, 1989; Townsend, 1972, 1974; Townsend \& Ashby, 1978; Townsend \& Nozawa, 1995; Townsend \& Schweickert, 1989), advances that allow for strong-inference tests of a complete set of hypotheses regarding these characteristics. We begin by considering the correspondence between the core assumptions of the dual-mode hypothesis and assumptions regarding four fundamental characteristics of information processing - process architecture, stopping rule, independence, and capacity (Townsend \& Ashby, 1983; Townsend \& Nozawa, 1995).

We begin with the issue of process architecture. Generally, there are three theoretical alternatives to consider. First, featural information may be processed first, followed by configural information (or vice versa) ${ }^{3}$ In this case, processing would be characterized as serial. Second, featural and configural information may be processed at the same time; in this case, processing would be characterized as parallel. Finally, featural and configural information may be initially processed in parallel, and the outputs of this processing may be pooled or combined into a single channel of information. In this case, processing would be characterized as a special form of parallel processing known as coactive processing (e.g., Diederich, 1995; Miller, 1982, 1991; Mordkoff \& Egeth, 1993; Mordkoff \& Yantis, 1991; Townsend \& Nozawa,
1995). According to the first assumption of the dualmode hypothesis, featural and configural information is simultaneously available, which maps naturally to an assumption of parallel processing.

The issue of a stopping rule refers to the amount of information that is required in order for a response to be selected. Two alternatives are generally considered. On the one hand, it may be that once some sufficient amount of either featural or configural evidence has accumulated, a response can be made. In this case, the stopping rule would be referred to as a self-terminating, or minimumtime, rule. On the other hand, it may be that there is some requisite amount of both featural and configural evidence required before a response can be made. In this case, the stopping rule would be referred to as an exhaustive, or maximum-time, rule. In addition to suggesting that the two sources of information are simultaneously available, the dual-mode hypothesis assumes that the orientation of the face determines which source will be dominant in processing. In particular, configural information is thought to dominate when the face is upright, with featural information dominating upon inversion (e.g., Leder \& Bruce, 2000). This assumption, in conjunction with the evidence used to support it, is consistent with the idea that the source of information that best supports a response is used, implying it is not necessary to have a requisite amount of both featural and configural information. Thus, the dual-mode hypothesis seems to require the assumption of a self-terminating, or minimum-time, stopping rule.

The issue of independence refers to the effect that the processing of one type of information may or may not have on the rate 4 at which another type of information is processed. The first of the two alternatives that need to be considered is that processing featural information has no effect on the rate at which configural information is processed, and vice versa. In this case, the rates of processing of the two sources of information would be characterized as independent. In contrast, it might be that configural information increases the rate at which featural information is processed or that featural information decreases the rate at which configural information is processed. In both of these cases, the rates of processing would be characterized as either positively or negatively dependent. As stated in the second assumption of the dual-mode hypothesis, configural information and featural information are thought to be independently processed. Consequently, we would expect to see no effect of the rate of processing for one source of information on the rate of processing for the other.

The issue of capacity is closely related to the issue of independence (Townsend \& Wenger, 2004b) and refers to the way in which system performance changes as workload is varied (for discussions, see Townsend \& Ashby, 1978; Townsend \& Nozawa, 1995; Townsend \& Wenger, 2004b; Wenger \& Townsend, 2000). For example, if it is assumed that when featural information is augmented by configural information (i.e., if the amount 
of information to be processed and, thus, the workload are increased) there will be no observable effect on processing efficiency, the perceptual system can be assumed to possess unlimited capacity. If, in contrast, it is assumed that augmenting one of these sources of information with the other will result in a decrement in performance, the assumption is one of limited-capacity processing. Finally, if this same change in workload produces an improvement in performance, the inference is supercapacity processing. If we use, as a starting point, the processing of inverted faces and contrast that with the processing of upright faces, the dual-mode hypothesis would characterize this change as an increase in the amount of configural information that is available, expressed by the third assumption of the hypothesis (Leder \& Bruce, 2000; Searcy \& Bartlett, 1996). Since, generally, performance with upright faces is expected to be better than performance with inverted faces, this would seem to suggest that increasing the amount of information produces an improvement in performance. As a consequence, the dual-mode hypothesis would seem to require the assumption of supercapacity processing. However, since both sources of information are available in upright and inverted faces, it seems safest to assume that processing must be either unlimited or supercapacity.

\section{Testing the Processing Hypothesis}

The discussion in the preceding section mapped the dual-mode hypothesis onto four hypotheses regarding the fundamental characteristics of information processing - specifically, (1) parallel, (2) independent processing of featural and configural information, using (3) a selfterminating (minimum-time) stopping rule, with (4) overall system performance being characterized in terms of unlimited or supercapacity processing. In developing the mapping between the dual-mode hypothesis and the four hypotheses regarding information processing, we also delineated a set of alternative hypotheses. ${ }^{5}$ We now will consider how we can subject this set of hypotheses to simultaneous, strong-inference, experimental tests.

Ideally, all of these hypotheses should be tested with the performance of a single observer. This, in fact, is possible, due to a set of methodological and theoretical accomplishments summarized most completely and recently by Townsend and Nozawa (1995). The experimental paradigm allowing for these possibilities-simultaneous strong-inference tests of a complete set of hypotheses, within the data for a single observer-is known as the double-factorial paradigm (see also Townsend \& Wenger, 2004a).

As its name implies, the double factorial paradigm consists of a set of nested $2 \times 2$ factorial designs. Figure 1 provides a schematic representation of the doublefactorial paradigm, as it was used in the present study. Other examples of the use of this paradigm include Townsend and Nozawa (1995), Townsend and Fific (2004), Hughes and Townsend (1998), and Wenger and Townsend (2001). For our purposes, the outer factorial involved the manipulation of featural and configural information. Specifically, observers were asked to determine, given a pair of images, whether the second of these images contained a change in features or a change in the configuration of the features. The inner factorial involved a manipulation designed to selectively influence the speed with which the featural and the configural information could be processed. Specifically, changes to either the featural or the configural information, when present, could be either ambiguous or unambiguous, with the former type of change assumed to be processed more slowly than the latter.

To be able to use the double-factorial paradigm, a minimum of two conditions need to be met (for more extensive discussions, see Townsend \& Wenger, 2004a; Wenger $\&$ Townsend, 2001). First, one must identify an experimental manipulation that can be assumed to exert selective influence (e.g., Dzhafarov, 1997) on the speed of processing each of the dimensions of interest (the featural and configural changes, in our case). Selective influence is required due to the fact that it is a critical assumption in the proofs (Townsend \& Nozawa, 1995) that relate the dependent measures to the inferences regarding processing characteristics (to be discussed momentarily). Second, since it is critical to be able to distinguish serial from parallel processing, we need to be sure that any evidence for seriality (if obtained) can be localized to perceptual processing. Consequently, the featural and configural information needs to be contained in an image sized so as to be apprehended in a single fixation and presented for a duration that disallows eye movements.

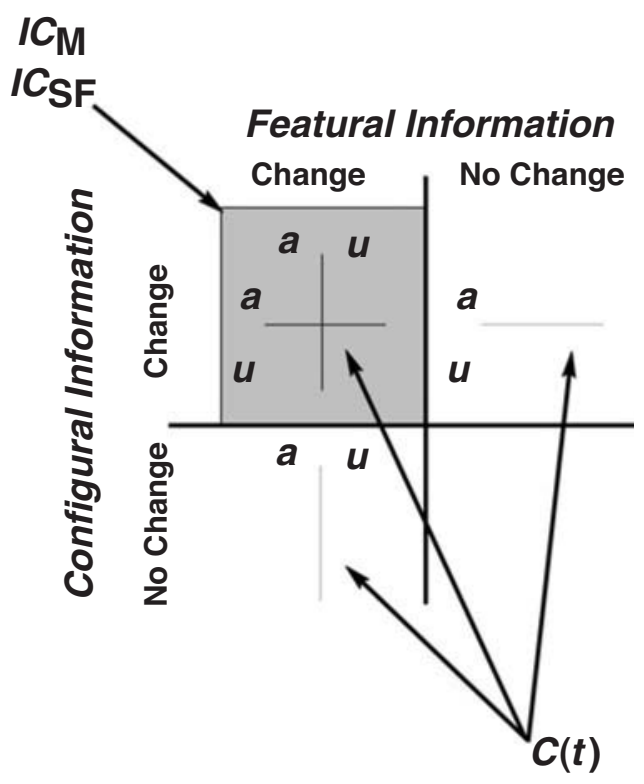

Figure 1. Schematic representation of the double-factorial paradigm as used in the present study. $I C_{\mathrm{M}}$, interaction contrast at the level of the mean; $I C_{\mathrm{SF}}$, interaction contrast at the level of the survivor function; $a$, ambiguous change; $\boldsymbol{u}$, unambiguous change; $C(t)$, capacity coefficient. See the text for details. 
As developed by Townsend and Nozawa (1995), the double-factorial paradigm provides for a range of data that can be used to simultaneously test, in the performance of individual observers, the four hypotheses regarding the fundamental characteristics of information processing described above. These tests require that the response times (RTs) from different portions of the overall design be used in various ways. We begin by considering the sources of evidence for inferences regarding the process architecture and the stopping rule. The data critical to these inferences are obtained in the upper right quadrant of the overall design (the grayed set of cells in Figure 1). Here, both sources of information are changed in the test stimulus, holding workload constant as processing speed is manipulated. To make inferences regarding the architecture and the stopping rule, we first estimate an interaction contrast, at the level of the mean RTs, for the $2 \times 2$ factorial involving the ambiguity manipulation:

$$
I C_{\mathrm{M}}=\overline{R T}_{\mathrm{AA}}-\overline{R T}_{\mathrm{AU}}-\overline{R T}_{\mathrm{U} \mathrm{A}}+\overline{R T}_{\mathrm{UU}} .
$$

Here, $\overline{R T}$ indicates the mean RT in one of the four cells of the inner factorial. The two subscripts in Equation 1 refer to the state of the featural and configural change, with an $\mathrm{A}$ indicating an ambiguous change and a $\mathrm{U}$ indicating an unambiguous change.

The second source of evidence for inferences regarding the architecture and the stopping rule is an interaction contrast calculated at the level of the survivor function, $S(t),{ }^{6}$ of the RT distribution:

$$
I C_{\mathrm{SF}}=S_{\mathrm{AA}}(t)-S_{\mathrm{UA}}(t)-S_{\mathrm{AU}}(t)+S_{\mathrm{UU}}(t) .
$$

Whereas the interaction contrast at the level of the mean (Equation 1) gives a single value, the interaction contrast at the level of the survivor function (Equation 2) gives a function for the range of values represented in the RT distribution. In a set of rigorous and distribution-free proofs in which the distribution of the base (or residual) times (e.g., motor execution times) was also considered, Townsend and Nozawa (1995) demonstrated that if both of these interaction contrasts are used, it is possible to make strong inferences regarding both the architecture and the stopping rule. Using either alone does not allow for this type of strong inference. Table 1 relates the possible outcomes for each of the interaction contrasts to the inferences regarding the architecture and the stopping rule. Readers interested in the formal analyses supporting this logic are encouraged to consult Townsend and Nozawa (1995, pp. 351-356). ${ }^{7}$

For example, imagine that the interaction contrast at the level of the mean (Equation 1) is found to be statistically indistinguishable from zero. Consulting Table 1, we see that although this outcome would falsify all forms of parallel processing, no inference would be possible regarding the stopping rule. Now imagine that the interaction contrast at the level of the survivor function (Equation 2), for the same data set, produced a function that was negative for the shortest RTs and positive for the longest RTs. It would then be possible to falsify the hypothesis of
Table 1

Possible Outcomes for Each of the Interaction Contrasts

\begin{tabular}{|c|c|c|c|}
\hline \multirow[b]{2}{*}{$I C_{\mathrm{M}}$} & \multirow[b]{2}{*}{$I C_{\mathrm{SF}}$} & \multicolumn{2}{|r|}{ Inferences } \\
\hline & & Architecture & Stopping Rule \\
\hline 0 & 0 & serial & self-terminating (minimum time) \\
\hline 0 & $-\rightarrow+$ & serial & exhaustive (maximum time) \\
\hline$>0$ & $>0$ & parallel & self-terminating (minimum time) \\
\hline$>0$ & $-\rightarrow+$ & coactive & \\
\hline$<0$ & $<0$ & parallel & exhaustive (maximum time) \\
\hline
\end{tabular}
(Equations 1 and 2) and the Inferences Supported

Note-The mapping between outcomes and inferences is formally established in Townsend and Nozawa (1995, pp. 351-356).

self-terminating (minimum-time) processing. Using both interaction contrasts would then lead to the unique (i.e., only allowable) conclusion that processing was performed serially, using an exhaustive stopping rule.

\section{Capacity and Independence}

Inferences regarding the architecture and the stopping rule require that the workload be held constant while the processing rate is varied. Inferences regarding capacity and independence are made by holding speed of processing constant across changes in workload; in the present study, this was done by keeping both sources of information unambiguous while modifying workload from detection of two changes to one. The first source of evidence used in making these inferences comes from what is known as the capacity coefficient:

$$
C(t)=\frac{H_{F C}(t)}{H_{F}(t)+H_{C}(t)} .
$$

The quantities in the numerator and denominator of Equation 3 represent the integrated hazard functions on the RT distribution:

$$
\begin{aligned}
H(t) & =\int_{t^{\prime}=0}^{t} h\left(t^{\prime}\right) d t^{\prime} \\
& =\int_{t^{\prime}=0}^{t} \frac{f\left(t^{\prime}\right)}{S\left(t^{\prime}\right)} d t^{\prime} .
\end{aligned}
$$

The subscripts in Equation 3 indicate the type of change involved: $\mathrm{F}$ for trials involving feature changes, $\mathrm{C}$ for trials involving configuration changes, and $\mathrm{FC}$ for trials involving both types of change. The importance of the integrated hazard function in making inferences regarding capacity comes from its interpretation. As has been discussed by Townsend and Ashby (1978), the (nonintegrated) hazard function on time can be interpreted in terms of instantaneous intensity - that is, the amount of work a system is capable of accomplishing in an instant of time. Its integrated form can then be interpreted in terms of the cumulative amount of work a system is capable of performing up to a particular point in time (for a tutorial discussion of these concepts, see Wenger \& Townsend, 2000).

The derivation and interpretation of the capacity coefficient (Equation 3 ) is based on an equality from proba- 
bility theory (see Townsend \& Ashby, 1983). Specifically, in terms of the processing hypotheses considered here, if one assumes parallel, independent processing, with a self-terminating (minimum-time) stopping rule, the integrated hazard function for the trials involving two changes should be equal to the sum of the integrated hazard functions for the two types of single-change trials. Stated in terms of cumulative work, this means that under the assumptions above, the amount of work that can be accomplished with two changes is equal to the sum of the work done in the two types of trials involving only one change. Conceptually, this maps nicely onto the notion of unlimited-capacity processing and would be indicated by having $C(t)$ take on the value of 1 . To the extent that having two types of changes present increases the ability of the perceptual system to work with the stimuli, we should expect $C(t)$ to exceed 1, with the naturally associated inference being supercapacity processing. Finally, to the extent that having two changes decreases the ability of the perceptual system to process the stimulus information, we should expect $C(t)$ to be below 1 , with the associated inference being limited-capacity processing.

Townsend and Nozawa (1995) formally demonstrated that the capacity coefficient is systematically related (but not identical) to two well-known inequalities in the RT literature. These relations essentially allow these inequalities to be used, with the capacity coefficient, as sources of converging evidence for inferences regarding capacity. The first of these inequalities originated with Miller (1982):

$$
S_{\mathrm{FC}}(t)-S_{\mathrm{F}}(t)-S_{\mathrm{C}}(t)+1 \geq 0 .
$$

We follow the presentation of Townsend and Nozawa and consider the inequality as it is expressed in terms of survivor functions, rather than the cumulative distribution functions (see note 6). A violation of this inequality would be consistent with extreme supercapacity processing and $C(t)$ values much greater than 1 .

The second inequality originated with Grice (Grice, Canham, \& Boroughs, 1984; Grice, Canham, \& Gwynne, 1984):

$$
\min \left[S_{\mathrm{F}}(t), S_{\mathrm{C}}(t)\right]-S_{\mathrm{FC}}(t) \geq 0 .
$$

This inequality serves a complementary function to that of the Miller inequality. Specifically, violation of the Grice inequality would be consistent with limited-capacity processing and a $C(t)$ value much less than $1 .{ }^{8}$

As was mentioned earlier, capacity and independence are very tightly related issues. Townsend and Wenger (2004b), using numerical simulations, have demonstrated that extreme (large and small) values of the capacity coefficient (Equation 3, along with an analogous form for exhaustive processing) are related to positive and negative channel dependencies. Thus, any inference of a violation of independence in our data should be suggested by, on the positive side, large values for $C(t)$, along with violations of the Miller inequality, or on the negative side, small values of $C(t)$, along with violations of the Grice inequality.

\section{Summary: Hypotheses and Predictions}

On the basis of the assumptions of the dual-mode hypothesis (e.g., Bartlett \& Searcy, 1993; Cabeza \& Kato, 2000; Searcy \& Bartlett, 1996), we would expect to obtain evidence suggesting a parallel, independent, selfterminating architecture, with performance consistent with unlimited to supercapacity processing. This would be evidenced by $I C_{\mathrm{M}}>0, I C_{\mathrm{SF}}>0, C(t)>1$, and possible violations of the Miller inequality. Note that this pattern of evidence would falsify all types of serial processing, any form of exhaustive processing, and any possibility of limitations in capacity, thereby falisfying other hypotheses. Note also that these are predictions that can be tested in the data of individual observers. Detection of configural changes should be best in upright faces, and inversion should affect the detection of these types of changes more than it should featural changes. Finally, to the extent that these ideas are specific to the processing of facial stimuli, we expect that this pattern will be found only with facial stimuli.

\section{METHOD}

\section{Participants}

A total of 4 individuals were recruited from the Notre Dame community. All had normal or corrected-to-normal vision and unencumbered use of their hands. The participants were compensated at the rate of $\$ 7$ per hour.

\section{Materials}

Four stimulus types were used: two human faces and two abstract face-like shapes. It was determined to use face-like schematics in lieu of other alternatives, such as houses (e.g., Tanaka \& Sengco, 1997), for several reasons. First, the schematic faces allowed us to equate internal geometry and the abstract identity of the elements we manipulated. Should no differences between these schematic faces and the actual faces be observed, it would suggest that obtained effects do not reflect any type of specialized processing for faces but may be more related to the internal geometry and abstract (image-independent) characteristics. ${ }^{9}$ Conversely, reliable differences between the two types of images would suggest that images of human faces may in some way, indeed, be special with respect to the functioning of the human visual system. Furthermore, there exists a critical confound in the use of certain non-face-like stimuli for comparison with actual faces. Specifically, using stimuli that do not contain the left-right symmetry possessed by human faces raises the question of any differences that might be attributed to the stimuli: Are the stimuli being treated differently by the observers due to the fact they are not faces or due to the fact that they are asymmetric? The use of objects that contain the symmetry found in faces, as well as other face-like properties, without actually being faces eliminates this confound.

The human faces were represented by photographs of two females, cropped at the neck, with hair, in full-frontal views, placed on a black background. They were obtained by taking digital pictures of volunteers from the Notre Dame community and then were edited in order to isolate the head and the neck on a black background. The dimensions of the frames surrounding the faces were $2 \mathrm{~cm}(\mathrm{~h}) \times 2 \mathrm{~cm}(\mathrm{v})$. When viewed at a fixed distance of $91 \mathrm{~cm}$, the images subtended $1.26^{\circ}$ of visual angle horizontally and vertically.

The abstract face-like shapes, or schematic images, were produced by placing a gray ellipse on a black background, with the gray-scale value for the ellipse being equal to the mean gray-scale value for the interior of the faces. Four elements were placed inside the ellipse, corresponding to the features of a human face. The first 
Table 2

Nine Variants Created for Each of the Two Faces and Each of the Two Schematic Images

\begin{tabular}{cccccc}
\hline & \multicolumn{3}{c}{ Color } & & \multicolumn{2}{c}{ Position } \\
\cline { 2 - 3 } \cline { 5 - 6 } Variant & Lips & Irises & & Mouth & Eyes \\
\hline 1 & $\mathrm{R}$ & $\mathrm{B}$ & & $\mathrm{C}$ & $\mathrm{C}$ \\
2 & $\mathrm{P}$ & $\mathrm{G}$ & & $\mathrm{C}$ & $\mathrm{C}$ \\
3 & $\mathrm{R}$ & $\mathrm{B}$ & & $\mathrm{UR}$ & $\mathrm{X}$ \\
4 & $\mathrm{P}$ & $\mathrm{G}$ & & $\mathrm{UR}$ & $\mathrm{X}$ \\
5 & $\mathrm{R}$ & $\mathrm{B}$ & & $1 / 2(\mathrm{CUR})$ & $1 / 2(\mathrm{CX})$ \\
6 & $\mathrm{P}$ & $\mathrm{G}$ & & $1 / 2(\mathrm{CUR})$ & $1 / 2(\mathrm{CX})$ \\
7 & $1 / 2(\mathrm{RP})$ & $1 / 2(\mathrm{BG})$ & & $\mathrm{C}$ & $\mathrm{C}$ \\
8 & $1 / 2(\mathrm{RP})$ & $1 / 2(\mathrm{BG})$ & & UR & $\mathrm{X}$ \\
9 & $1 / 2(\mathrm{RP})$ & $1 / 2(\mathrm{BG})$ & & $1 / 2(\mathrm{CUR})$ & $1 / 2(\mathrm{CX})$ \\
\hline
\end{tabular}

Note- R, red; P, pink; B, blue; G, green; 1/2(RP), RGB midpoint for red and pink; 1/2(BG), RGB midpoint for blue and green; C, centered; UR, moved up and to the right; $X$, crossed; $1 / 2$ (CUR), midpoint between centered and moved up and to the right; $1 / 2(\mathrm{CX})$, midpoint between centered and crossed. See Table 3 for the RGB values for each of the four colors

Table 3

RGB Values Used for the Colors of the Eyes and Lips in the Face Stimuli and for the Corresponding Features in the Schematic Stimuli

\begin{tabular}{lrrr}
\hline Color & R & G & B \\
\hline Red & 255 & 51 & 77 \\
Blue & 99 & 198 & 248 \\
Pink & 225 & 51 & 121 \\
Green & 99 & 235 & 248 \\
\hline
\end{tabular}

of these was a small light gray circle placed near the center of the ellipse, in the position of a nose. Two distinct identities for these schematics were created by using ellipses in the positions of the eyes for one form and rectangles in the corresponding positions for the other form. The form of the pupils corresponded to the outer eye shape. In both schematics, rectangles were used in the position of the mouth. The frames containing these schematic images had the same dimensions as those containing the faces.

After all these images had been created, they were altered to produce nine variations (see Table 2). The first two of these variations were obtained by coloring (1) the lips red and the irises blue or (2) the lips pink and the irises green. RGB values used for each of these colors are presented in Table 3 . The corresponding features were colored in the same way in the schematics. The configural changes were made by crossing the eyes (by moving the pupils) and by rotating the mouth slightly up and to the right. This pair of changes corresponded to a change in facial expression that appeared natural to both the authors. Ambiguous featural changes were made by finding the midpoint (in terms of RGB values) between the two colors of the irises and the two colors of the lips. Ambiguous configural changes were made by reducing the magnitude of the shifts in the pupils and the mouth to halfway between the configural alteration and an unaltered face. Figure 2 presents examples of one of the faces and one of the schematics, with and without an unambiguous configural change.

An important methodological point needs to be made in regard to the configural manipulation. As was mentioned in the introduction, a configural change is any change that adjusts the spatial relationships among the features, or the face's internal geometry. In our case, this manipulation involved the pupils of the eyes and the position of the mouth. Typically, configural changes have been made by changing the physical location of the features, resulting in a very bizarre appearance for the configurally altered faces (e.g., Faulkner, Rhodes, Palermo, Pellicano, \& Ferguson, 2002; Searcy \& Bartlett, 1996; Webster \& MacLin, 1999), due in no small part to the fact that such changes are (excepting the effects of surgery or injury) physically impossible in actual faces. Due to the strange appearance of these configural manipulations, changes might be more obvious and might aid detection. Both of these problems - bizarreness and physical impossibility - suggested that we needed to use a configural manipulation that was both natural in appearance (e.g., a change in expression) and physically possible in normal faces. We should note, however, that although our manipulations were not the
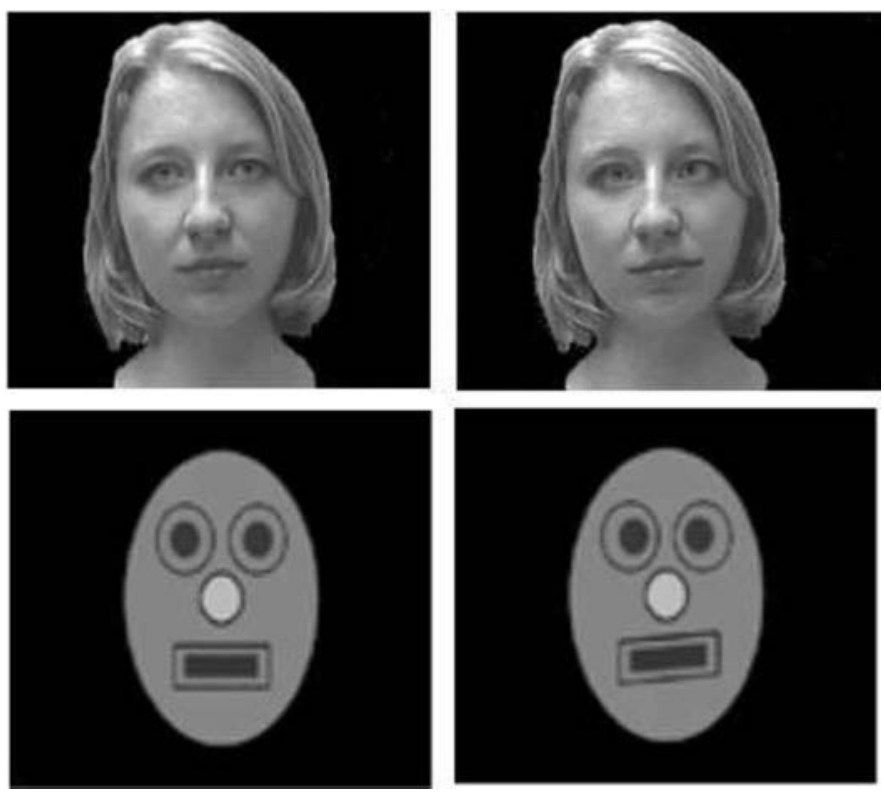

Figure 2. Example face (top row) and schematic (bottom row) stimuli, before (left column) and after (right column) an unambiguous configural change. 
same as those in earlier studies, they still resulted in a modification of the spatial relationships among the features and, thus, adhered to the definition employed in all the relevant studies.

All the images were presented at an intensity of $1 \mathrm{~cd} / \mathrm{m}^{2}$ (measured for the average gray-scale value in the images), using tachistoscopic LCD goggles (Milgram, 1997; Wenger, Hill, \& Klatt, 2001). ${ }^{10}$ The images were presented on a $43-\mathrm{cm}$ (diagonal) monitor, set to a resolution of $800 \times 600$ pixels $(26$ pixels $/ \mathrm{cm})$. Responses were made using a custom response box; measured RTs were accurate to $\pm 1 \mathrm{msec}$ (Forster \& Forster, 2003).

\section{Design and Procedure}

The experiment was conducted as a 2 (object type: face or schematic) $\times 2$ (orientation: upright or inverted) $\times 2$ (featural change: present or absent) $\times 2$ (configural change: present or absent) factorial design. For those cells of the design that involved a featural change, a configural change, or both, there was an additional manipulation of the magnitude of the change (unambiguous or ambiguous). All the factors were manipulated within observers. (See Figure 1 for a diagrammatic representation of the experimental design.)

The observers participated in 1 session every day for 5 weeks, resulting in approximately 35 sessions for each participant, less some for illness or other absences. Each session lasted approximately $1 \mathrm{~h}$. A session started with 5 min of dark adaptation to maximize visual acuity. Following dark adaption, four blocks were presented; each block consisted of 96 trials. Of the four blocks, two presented all the stimuli in an upright, or normal, orientation, and the other two presented all the stimuli in an inverted orientation. Trial frequencies within each block were set so as to negate contingency benefits for the two-change conditions (see Mordkoff \& Yantis, 1991; Townsend \& Nozawa, 1995; Wenger \& Townsend, 2001).

Each trial began with the presentation of a fixation cross for $500 \mathrm{msec}$. Following this, the study stimulus was presented for $500 \mathrm{msec}$. The study stimulus could be any one of the nine variants (see Table 2) of either one of the faces or one of the schematics. The orientation of the stimulus depended on the block, either upright or inverted, as was mentioned above. The screen was then cleared and remained blank for an interstimulus interval whose duration was uniformly distributed between 400 and $500 \mathrm{msec}$. The test stimulus was then presented for $75 \mathrm{msec}$. The test stimulus was of the same identity as the study stimulus and could vary configurally, featurally, in both ways, or not at all; these changes could be ambiguous or unambiguous. The participants were instructed to give a positive response (using the index finger of their dominant hand) if they perceived any change and a negative response (using the index finger of their nondominant hand) if they did not perceive any change. Blocks contained equal numbers of trials containing changes and trials without changes. Following the observer's response, feedback as to the correctness of the response was presented for $500 \mathrm{msec}$.

\section{RESULTS}

The first 15 blocks of trials for each observer were discarded as practice; for the remaining blocks, only those trials that elicited a correct response were included in the analyses reported below. We will begin by considering the data with respect to the assumption of selective influence. We then will examine the measures that support inferences regarding the architecture and the stopping rule and then those pertinent to capacity and independence. Finally, we will consider the effects of inversion. Error rates in all cases were less than 4\%; because of the low rate and because all the predictions concern RT data, we will report no analyses of the accuracy data. Prior to analyses, any latencies of less than $100 \mathrm{msec}$ or greater than 2,000 msec were discarded as reflecting anticipatory responses or lapses of attention. Initial analyses indicated that there were no reliable effects due to the specific face or schematic. Consequently, all the analyses reported below are collapsed across the two faces and the two schematics.

\section{Selective Influence}

The assumption of selective influence is critical to the interpretation of the interaction contrasts (Equations 1 and 2) that support inferences regarding architecture and the stopping rule (Townsend \& Nozawa, 1995). The assumption applies to the manipulation used to influence the processing speed for each of the two changes. Thus, the data that are of interest are those from the upper left quadrant of Figure 1.

We will begin by examining these data at the level of the mean for evidence consistent with the assumption

Table 4

Mean Response Times (in Milliseconds) as a Function of Ambiguity for All Observers $\left(\mathrm{O}_{1}-\mathrm{O}_{4}\right)$ When Both Sources Are Changed

\begin{tabular}{|c|c|c|c|c|c|c|c|c|c|}
\hline \multirow[b]{2}{*}{ Configuration } & \multirow[b]{2}{*}{ Feature } & \multicolumn{4}{|c|}{ Upright Stimuli } & \multicolumn{4}{|c|}{ Inverted Stimuli } \\
\hline & & $\mathrm{O}_{1}$ & $\mathrm{O}_{2}$ & $\mathrm{O}_{3}$ & $\mathrm{O}_{4}$ & $\mathrm{O}_{1}$ & $\mathrm{O}_{2}$ & $\mathrm{O}_{3}$ & $\mathrm{O}_{4}$ \\
\hline \multicolumn{10}{|c|}{ Faces } \\
\hline \multirow[t]{2}{*}{ Ambiguous } & Ambiguous & 496 & 647 & 530 & 482 & 514 & 631 & 530 & 465 \\
\hline & Unambiguous & 433 & 563 & 447 & 410 & 451 & 564 & 435 & 404 \\
\hline \multirow[t]{2}{*}{ Unambiguous } & Ambiguous & 437 & 511 & 460 & 404 & 440 & 536 & 458 & 393 \\
\hline & Unambiguous & 407 & 501 & 434 & 391 & 419 & 531 & 403 & 383 \\
\hline \multirow[t]{2}{*}{$I C_{\mathrm{M}}$} & & 33 & 74 & 57 & 59 & 42 & 62 & 40 & 51 \\
\hline & \multicolumn{9}{|c|}{ Schematics } \\
\hline \multirow[t]{2}{*}{ Ambiguous } & Ambiguous & 447 & 540 & 499 & 431 & 470 & 552 & 505 & 395 \\
\hline & Unambiguous & 385 & 451 & 446 & 363 & 396 & 461 & 439 & 341 \\
\hline \multirow[t]{2}{*}{ Unambiguous } & Ambiguous & 364 & 442 & 417 & 347 & 370 & 455 & 441 & 322 \\
\hline & Unambiguous & 351 & 424 & 403 & 315 & 353 & 423 & 430 & 305 \\
\hline$I C_{\mathrm{M}}$ & & 49 & 71 & 39 & 36 & 57 & 59 & 55 & 37 \\
\hline
\end{tabular}


Table 5

Results of the ANOVA, Double-Target Trials, for All 4 observers $\left(\mathrm{O}_{1}-\mathrm{O}_{4}\right)$

\begin{tabular}{|c|c|c|c|c|c|c|c|c|c|}
\hline \multirow{2}{*}{ Ambiguity } & & \multicolumn{4}{|c|}{ Upright Stimuli } & \multicolumn{4}{|c|}{ Inverted Stimuli } \\
\hline & & $\mathrm{O}_{1}$ & $\mathrm{O}_{2}$ & $\mathrm{O}_{3}$ & $\mathrm{O}_{4}$ & $\mathrm{O}_{1}$ & $\mathrm{O}_{2}$ & $\mathrm{O}_{3}$ & $\mathrm{O}_{4}$ \\
\hline \multicolumn{10}{|c|}{ Faces } \\
\hline Configural (C) & $F$ & $44.92 * * *$ & $72.95 * * *$ & $21.00 * * *$ & $28.73 * * *$ & $66.87 * * *$ & $26.72 * * *$ & $34.76 * * *$ & $31.65 * * *$ \\
\hline Featural $(\mathrm{F})$ & $F$ & $54.68 * * *$ & $16.38 * * *$ & $34.88 * * *$ & $22.85^{* * *}$ & $42.53 * * *$ & $8.27 * *$ & $70.77 * * *$ & $18.54 * * *$ \\
\hline $\mathrm{F} \times \mathrm{C}$ & $F$ & $7.17 * *$ & $10.35^{* *}$ & $9.67 * *$ & $10.64 * *$ & $10.13^{* *}$ & $6.22 *$ & $5.06^{*}$ & $9.38 * *$ \\
\hline & $M S_{\mathrm{e}}$ & $7,404.74$ & $28,869.91$ & $13,851.84$ & $14,852.29$ & $7,721.70$ & $32,644.52$ & $12,544.87$ & $12,711.13$ \\
\hline \multicolumn{10}{|c|}{ Schematics } \\
\hline $\mathrm{C}$ & $F$ & $148.53 * * *$ & $32.03 * * *$ & $76.93 * * *$ & $68.51 * * *$ & $343.18 * * *$ & $36.07 * * *$ & $29.40 * * *$ & $66.59 * * *$ \\
\hline $\mathrm{F}$ & $F$ & $58.75^{* * *}$ & $23.97 * * *$ & $21.52 * * *$ & $39.41 * * *$ & $135.85^{* * *}$ & $30.12 * * *$ & $33.52 * * *$ & $28.69 * * *$ \\
\hline \multirow[t]{2}{*}{$\mathrm{F} \times \mathrm{C}$} & $F$ & $25.74 * * *$ & $10.26^{* *}$ & $7.53 * *$ & $4.91 *$ & $53.26^{* *}$ & $6.99 * *$ & $16.35 * * *$ & $7.41 * *$ \\
\hline & $M S_{\mathrm{e}}$ & $123,748.76$ & $29,156.86$ & $9,416.77$ & $13,799.44$ & $3,203.50$ & $31,039.16$ & $8,457.80$ & $10,141.21$ \\
\hline
\end{tabular}

Note-The numerator degrees of freedom for all effects is $1 . \quad * p<.05 . \quad * * p<.01 . \quad * * * p<.001$.

that the ambiguity manipulation selectively slowed processing for each of the change types. This was done by analyzing the correct RTs from trials involving both featural and configural changes, using a 2 (feature change: unambiguous or ambiguous) $\times 2$ (configuration change: unambiguous or ambiguous) analysis of variance (ANOVA), with analyses conduced separately for

Obs. 1: Faces, Upright
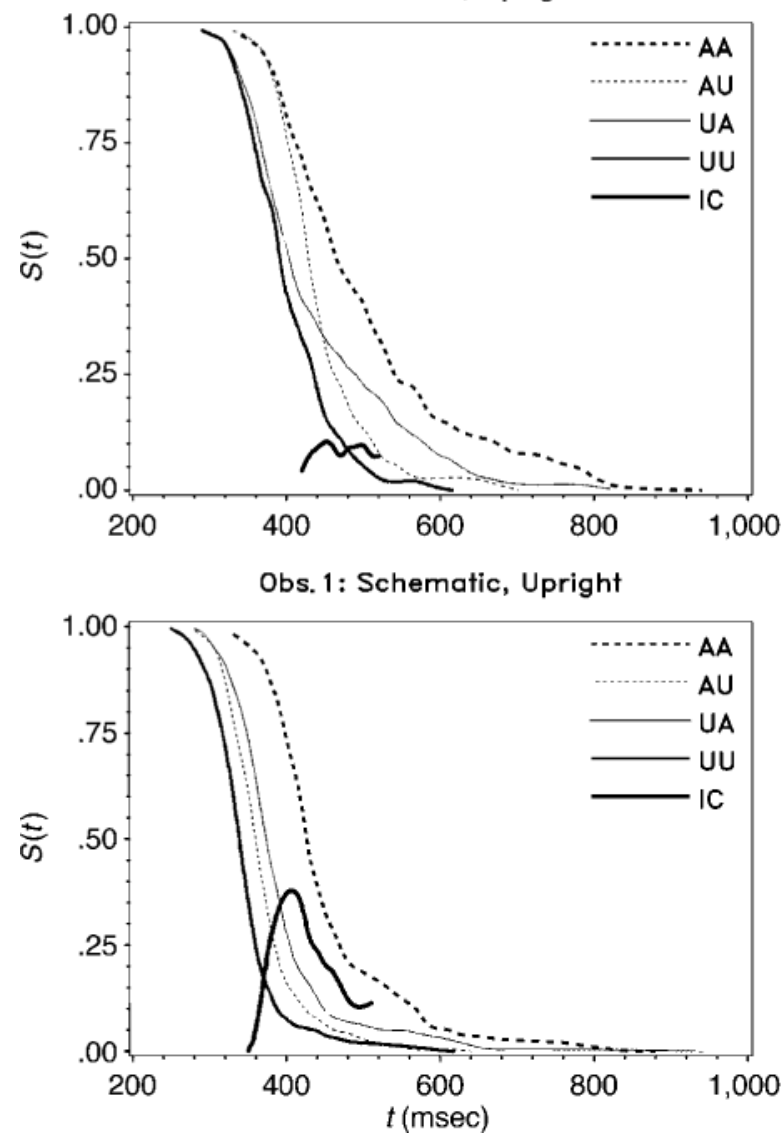

each observer. The mean RTs pertinent to this analysis are presented in Table 4, and the results of the ANOVAs are presented in Table 5. Evidence consistent with the assumption of selective influence, at the level of the mean, would take the form of main effects for each type of change, with ambiguous changes possessing longer RTs than unambiguous changes did. ${ }^{11}$ As can be seen in

Obs. 1: Faces, Inverted

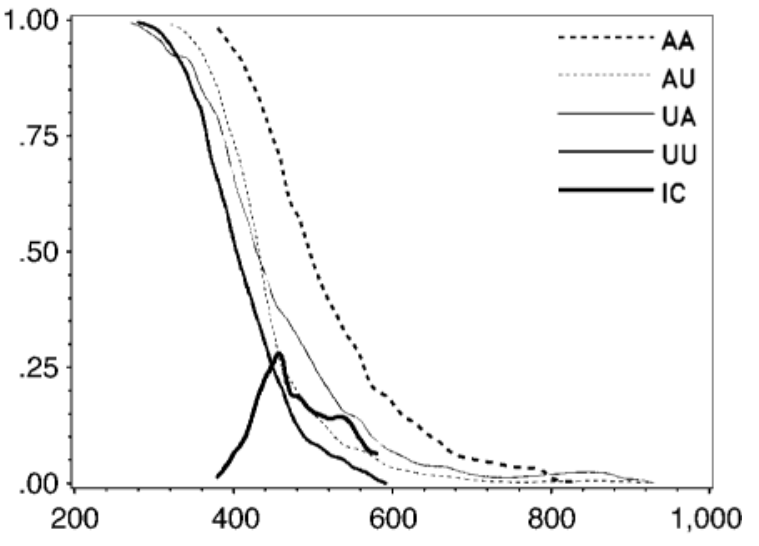

Obs. 1: Schematic, Inverted

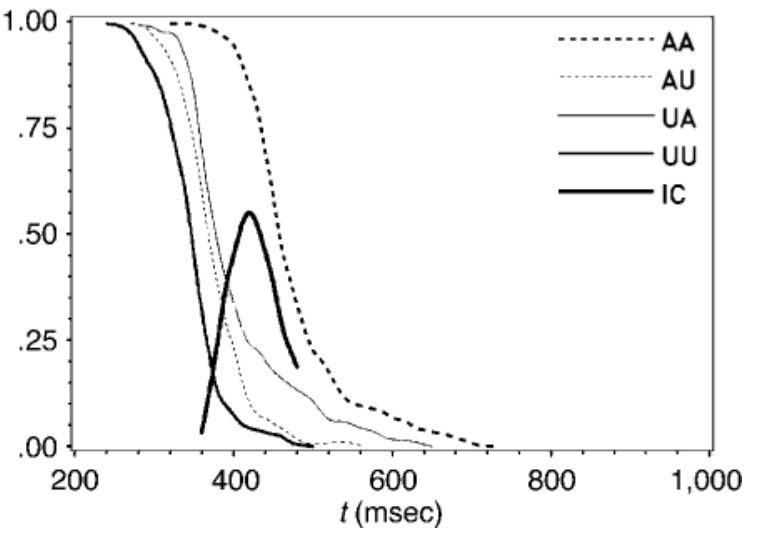

Figure 3. Observer 1: Survivor functions for the double-target trials, along with the interaction contrast $I C_{\mathrm{SF}}($ Equation 2). Values for this contrast are presented the range of times that are included in the central $95 \%$ of all of the component distributions. A, ambiguous change; $U$, unambiguous change. 
Table 5, all of the main effects were reliable for all stimulus types in both orientations, across all 4 observers, with the mean RTs for ambiguous changes being uniformly longer than those for unambiguous changes (see Table 4). Thus, it does appear that the data, at the level of the mean, are consistent with the assumption of selective influence.

The other level at which it is critical to assess the extent to which the data are consistent with the assumption of selective influence is the level of the survivor function of the RT distribution. Figures 3-6 present estimates of the survivor functions for each of the trials involving two changes; each panel of each figure corresponds to a different combination of stimulus class and orientation, for each of the 4 observers. Since $S(t)=P(T>t)$, higher values of $S(t)$ correspond to longer RTs. Thus, if the data are consistent with the assumption of selective influence at this level, the survivor functions for the trials involving two ambiguous changes should be above and to the right of the survivor functions for trials involving two unambiguous trials, with the survivor functions for the mixed trials being between the two. As can be seen in Figures 3-6, this pattern was observed for both stimulus types, in both orientations, across all 4 observers. This ordering was checked using Kolmogorov-Smirnov tests, and the results are presented in Table 6 . As can be seen in this table, all of the survivor functions were reliably ordered. ${ }^{12}$ Thus, the data appear to be consistent with the assumption of selective influence at the level of the survivor function, as well as at the level of the mean.

\section{Architecture and Stopping Rule}

Having established that the data are consistent with the assumption of selective influence, we can now consider the results pertinent to the inferences regarding the architecture and the stopping rule. Specifically, we can examine the interaction contrasts for the trials involving both featural and configural changes for evidence supporting the hypothesis of parallel self-terminating (minimumtime) processing. As a reminder, this hypothesis predicts that $I C_{\mathrm{M}}>0$ and $I C_{\mathrm{SF}}>0$. These outcomes, if obtained, would falsify any form of serial processing, and any form of exhaustive processing. Any other outcome would falsify parallel self-terminating (minimum-time) processing and would, thus, falsify the assumptions of the dual-mode hypothesis at this level.
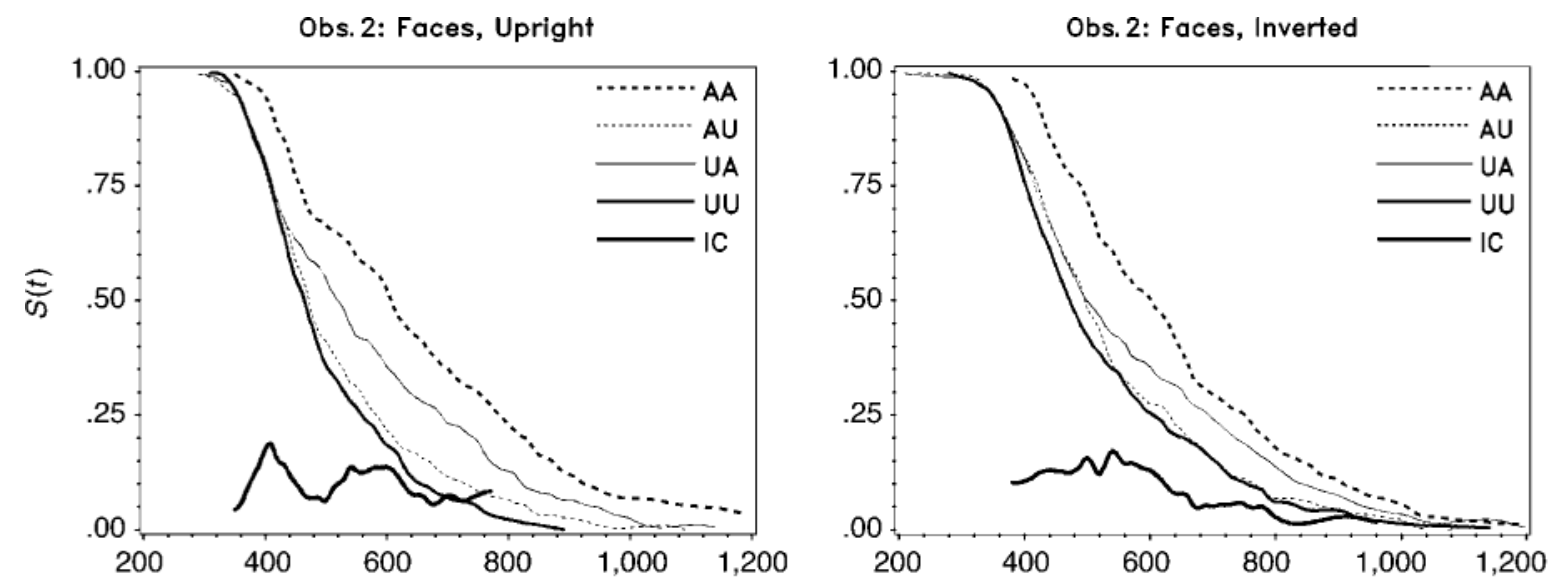

Obs. 2: Schematic, Upright

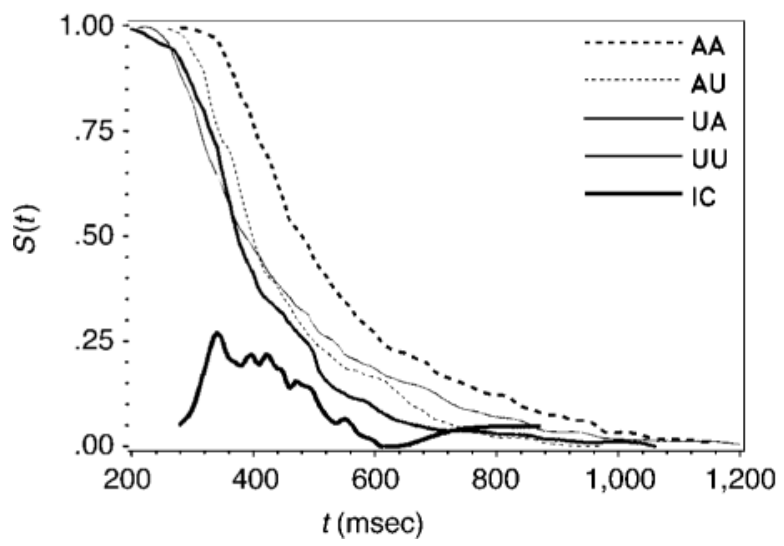

Obs. 2: Schematic, Inverted

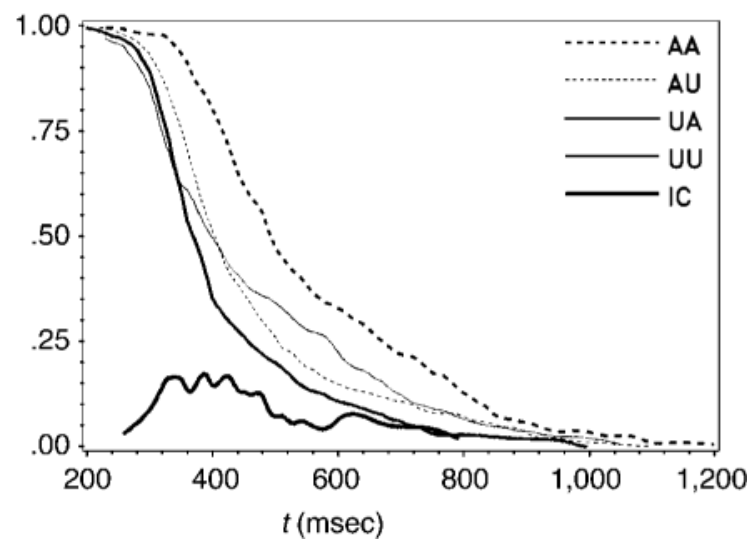

Figure 4. Observer 2: Survivor functions for the double-target trials, along with the interaction contrast $I C_{\mathrm{SF}}($ Equation 2). Values for this contrast are presented the range of times that are included in the central $95 \%$ of all of the component distributions. A, ambiguous change; $U$, unambiguous change. 
Obs. 3: Faces, Upright

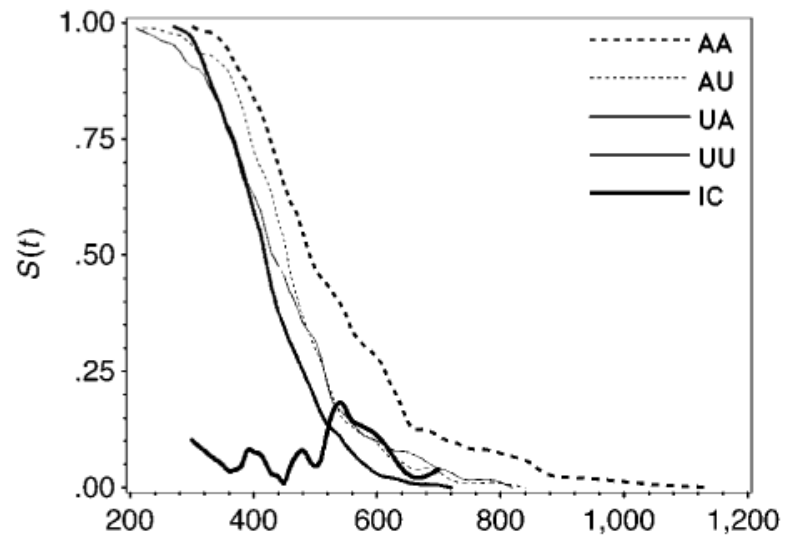

Obs, 3: Schematic, Upright

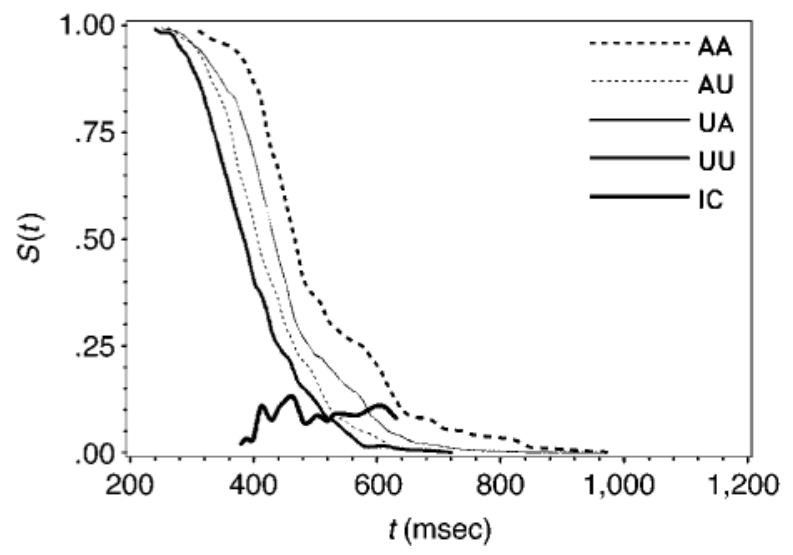

Obs. 3: Faces, Inverted

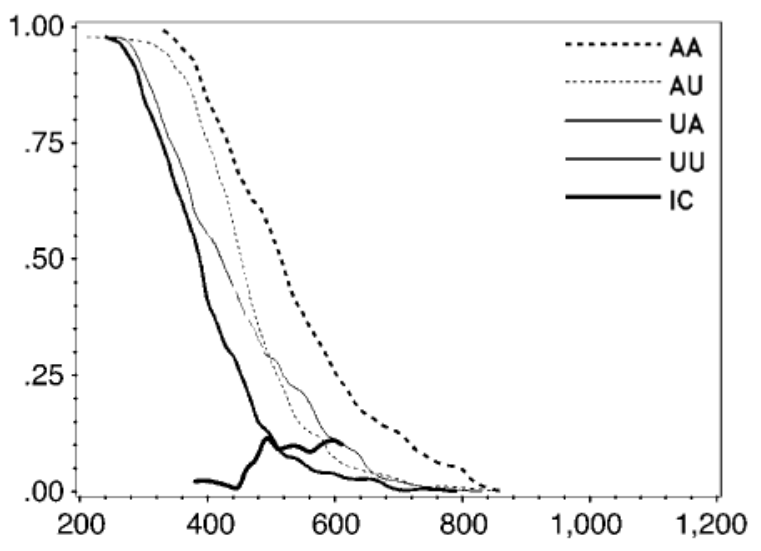

Obs, 3: Schematic, Inverted

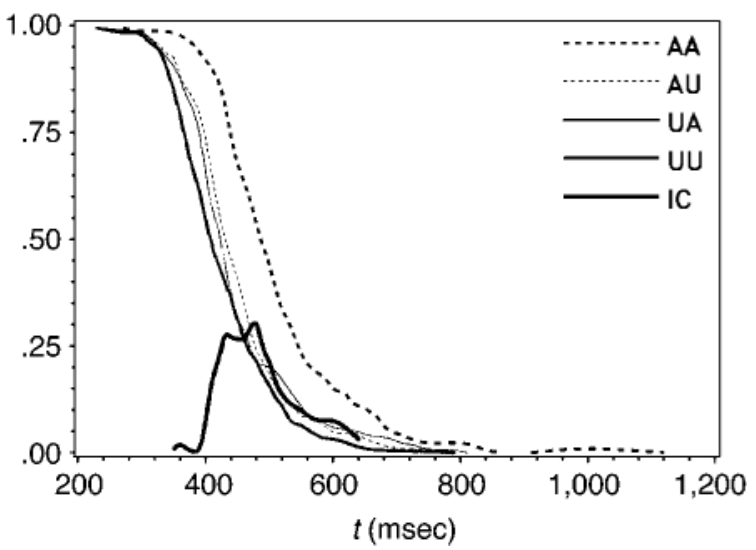

Figure 5. Observer 3: Survivor functions for the double-target trials, along with the interaction contrast $I C_{\mathrm{SF}}($ Equation 2). Values for this contrast are presented the range of times that are included in the central $95 \%$ of all of the component distributions. A, ambiguous change; $U$, unambiguous change.

The values of $I C_{\mathrm{M}}$ are presented in Table 4 , and the tests of the reliability of those interactions are presented in Table 5. As can be seen in these tables, all of the interaction contrasts were reliably positive. Thus, at this level of analysis, the data are consistent with the assumption of parallel self-terminating (minimum-time) processing. However, a reliably positive mean interaction contrast is also consistent with coactive processing (see Table 1). The interaction contrasts for the survivor functions can resolve this ambiguity. The values of this contrast are plotted as the thick dark lines in Figures 3-6. The contrasts were positive for both types of stimuli in both orientations across all 4 observers. Thus, the two interaction contrasts together (1) falsify all serial hypotheses and (2) unambiguously support the conclusion that the observers were processing these stimuli in a parallel manner, using a self-terminating (minimum-time) stopping rule, as predicted by the dual-mode hypothesis.

\section{Capacity and Independence}

The data critical for inferences regarding capacity and independence come from the cells of the design that in- volve a change in workload, holding processing speed for each change constant (see Figure 1). According to the dual-mode hypothesis, we would expect to see values for the capacity coefficient (Equation 3) that are greater than or equal to 1 , with accompanying violations of the Miller inequality (Equation 4). Any other outcomes would invalidate the dual-mode hypothesis at this level of analysis.

Figures 7-10 plot the values of $C(t)$ for both types of stimuli in both orientations for all 4 observers. The left

Table 6

Kolmogorov-Smirnov Test Statistics for Orderings on the Four Response Time Distributions Involving Both Featural and Configural Changes, as a Function of Object Type and Orientation, For All 4 Observers $\left(\mathrm{O}_{1}-\mathrm{O}_{4}\right)$.

\begin{tabular}{lccccc}
\hline Object & Orientation & $\mathrm{O}_{1}$ & $\mathrm{O}_{2}$ & $\mathrm{O}_{3}$ & $\mathrm{O}_{4}$ \\
\hline Face & Upright & $3.95^{* *}$ & $3.86^{* *}$ & $2.77^{* *}$ & $4.81^{* *}$ \\
& Inverted & $4.74^{* *}$ & $3.07^{* *}$ & $4.13^{* *}$ & $4.07^{* *}$ \\
Schematic & Upright & $7.55^{* *}$ & $4.36^{* *}$ & $4.57^{* *}$ & $5.76^{* *}$ \\
& Inverted & $9.50^{* *}$ & $4.92^{* *}$ & $4.39^{* *}$ & $5.28^{* *}$ \\
\hline
\end{tabular}

${ }^{* *} p<.01$ 
Obs. 4: Faces, Upright

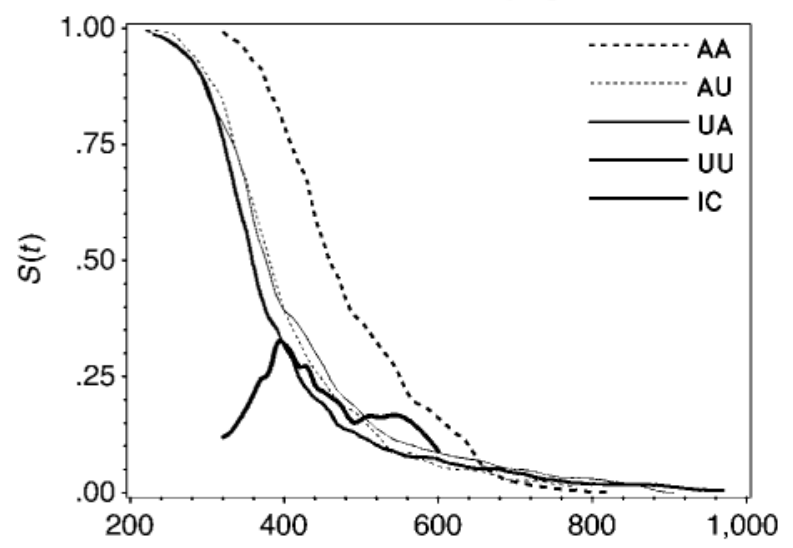

Obs. 4: Schematic, Upright

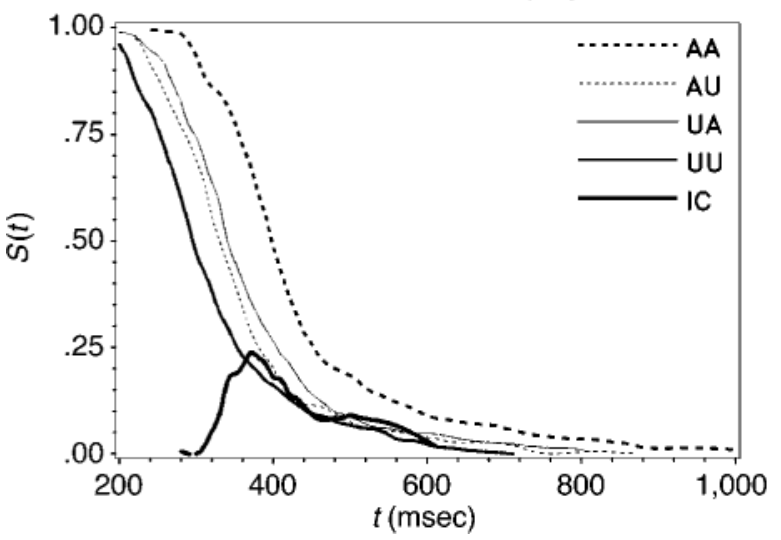

Obs. 4: Faces, Inverted
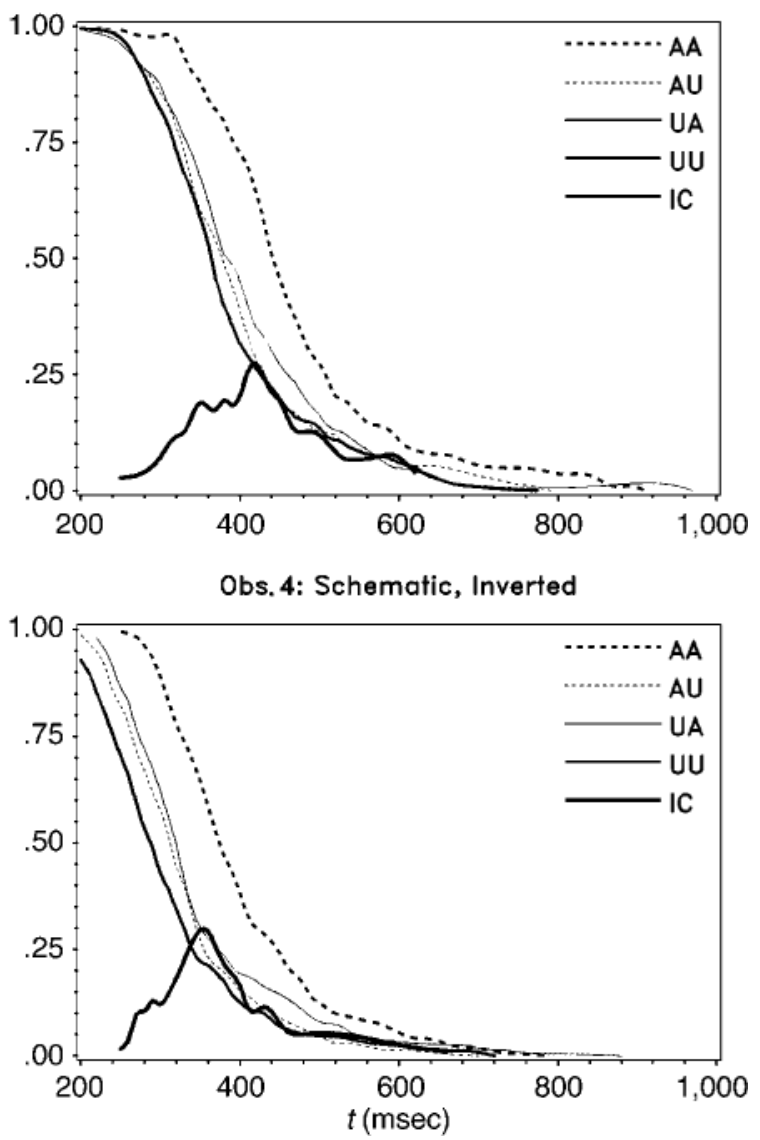

Figure 6. Observer 4: Survivor functions for the double target trials, along with the interaction contrast $I C_{\mathrm{SF}}($ Equation 2$)$. Values for this contrast are presented the range of times that are included in the central $95 \%$ of all of the component distributions. A, ambiguous change; $U$, unambiguous change.

ordinate axis in each panel of these figures represents the range of values of $C(t)$, and the dark thick line in each panel gives the obtained values for $C(t)$. The first point to note is that, generally, all values of $C(t)$ were greater than or equal to 1 , as would be expected on the basis of the dual-mode hypothesis. Slight deviations from this were obtained in the left- and right-hand tails of the distributions for Observers 2 and 4. Examination of the distributions for these 2 observers in those particular cases suggests that these deviations were due to extremely small numbers of observations in at least one of the component distributions contributing to Equation 3 and, thus, should not be interpreted as contradicting the overall pattern. A second point of note is that there were cases, particularly for Observers 1 and 2, where the relative capacity for processing the schematic images seemed greater than the relative capacity for processing faces. This is consistent with a pattern observed in a facial feature detection task reported by Wenger and Townsend (2001), where nonface stimuli did, in some cases, produce higher levels of capacity than did face stimuli.
A reviewer of an earlier version of this article correctly noted that the estimate of $C(t)$ may well be biased, depending on assumptions regarding base times. Under particular assumptions about base times in the singleand double-target trials, it is possible for the estimated value of $C(t)$ to be systematically below the true value. There are three issues that seem important with respect to this possibility. First, the specific assumptions regarding processing that would lead to a conservative bias themselves raise interesting questions; we will illustrate a subset of these in the appendix. Second, the values of $C(t)$ observed in this study all tend toward unlimitedcapacity to supercapacity processing; if there is a conservative bias in our estimates, it suggests that most or all of our observers evidenced supercapacity processing, an inference which is generally consistent with our current inferences. Finally, as has been noted by numerous authors (including Townsend \& Ashby, 1983; Townsend \& Nozawa, 1995; Townsend \& Wenger, 2004a, 2004b), the fact that a large segment of the literature on the modeling of RTs ignores the potential influences of base 
Obs. 1: Faces, Upright

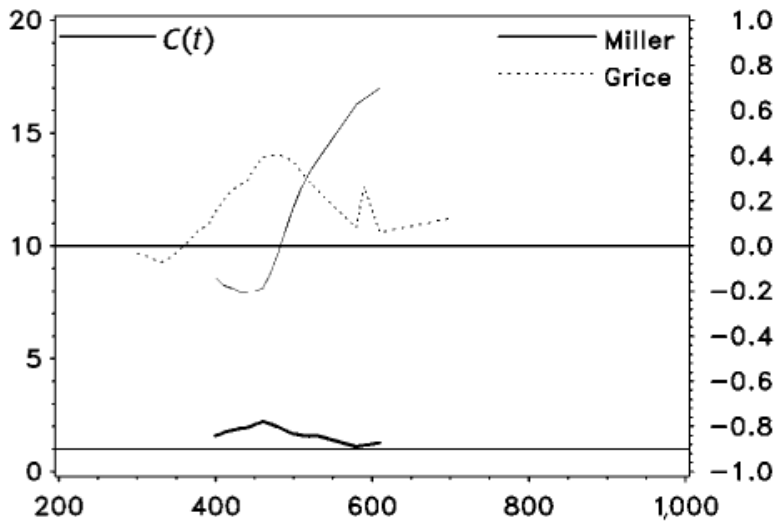

Obs. 1: Schematic, Upright

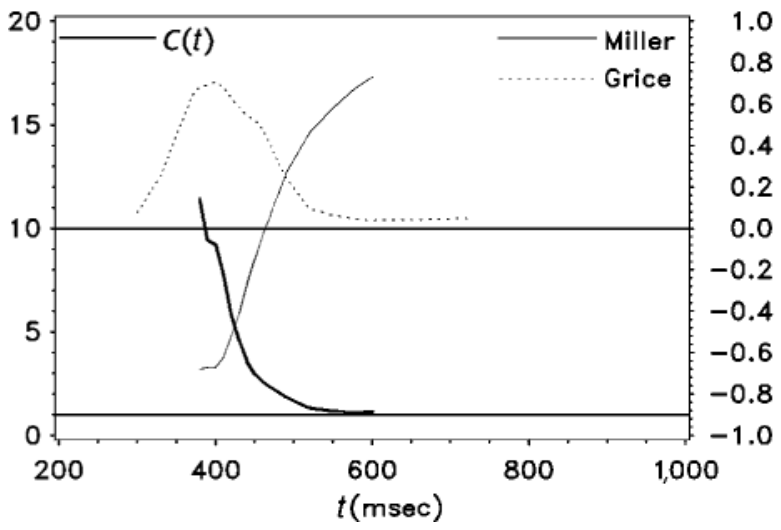

Obs. 1: Faces, Inverted

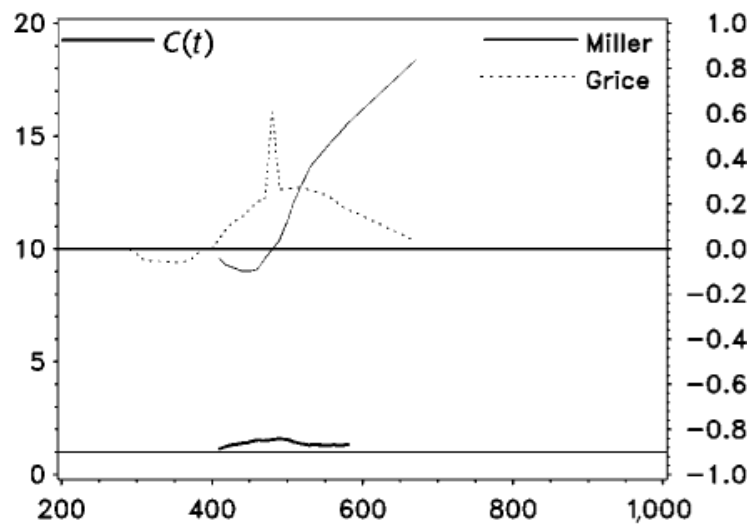

Obs. 1: Schematic, Inverted

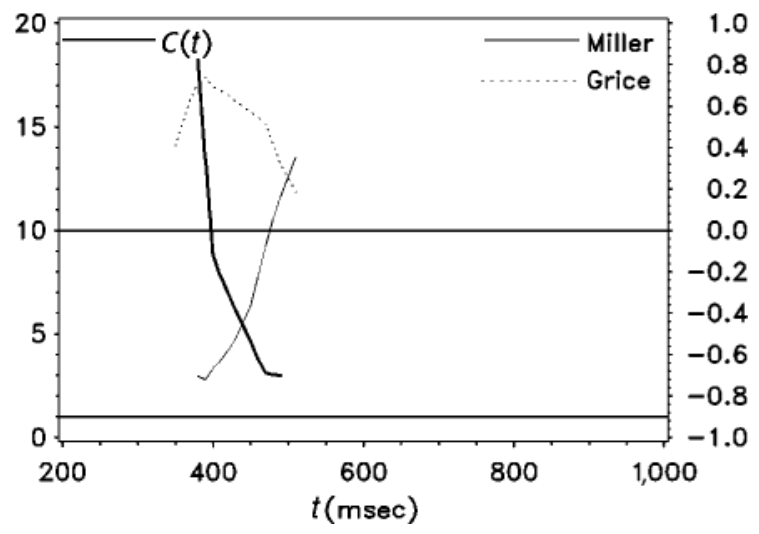

Figure 7. Observer 1: Capacity coefficients (Equation 3) and values of the Miller (Equation 4) and the Grice (Equation 5) inequalities, for the two stimulus classes in each of the two orientations.

times is both a major challenge and a major weakness. Although we do not wish to sidestep this important question, we do believe that a complete consideration of this complex issue goes well beyond the boundaries of the present study.

Figures 7-10 also present the values of the Miller (Equation 4) and the Grice (Equation 5) inequalities. The range of values for these inequalities is presented on the right ordinate of each panel of these figures, and the observed values of the inequalities are graphed as the thin solid and dashed lines. A violation of either inequality would be indicated by a value less than zero. As would be expected from the $C(t)$ data (see Townsend \& Nozawa, 1995; Townsend \& Wenger, 2004b), there were violations of the Miller inequality in those regions of the RT distribution where $C(t)$ was above one. The handful of violations of the Grice inequality all occurred in regions of the RT distribution where there were very few observations in one or more of the component distributions. Thus, the results from the Miller and the Grice inequalities support the inference of unlimited-capacity to supercapacity processing, for both faces and schematic images, when presented upright and inverted, for all 4 observers.
With respect to conclusions regarding independence, the magnitude of the $C(t)$ values are within ranges observed in other applications of the double-factorial paradigm (see, in particular, Townsend \& Nozawa, 1995; Wenger \& Townsend, 2001) and are below values observed in explorations of the effects of channel dependency on capacity (Townsend \& Wenger, 2004b); the evidence in favor of supercapacity processing thus requires some mechanism for producing these effects. The most plausible mechanism for these effects is a positive dependence in the rates of processing the featural and the configural information (J. T. Townsend, personal communication, July 26, 2002). Thus, it seems reasonable to conclude that there is some positive dependency at work. This, of course, suggests that the data contradict the assumptions of the dualmode hypothesis, at this level of analysis.

\section{Orientation Effects}

The results presented so far address hypothesized processing characteristics and provide strong support for three of the four assumptions of the dual-mode hypothesis, while allowing alternative hypotheses to be rejected. Specifically, the data unambiguously support self-terminating, 
Obs. 2: Faces, Upright

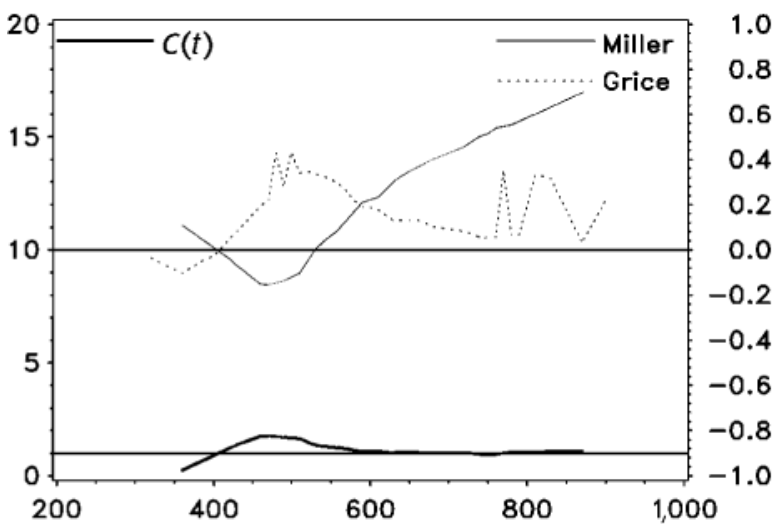

Obs. 2: Schematic, Upright

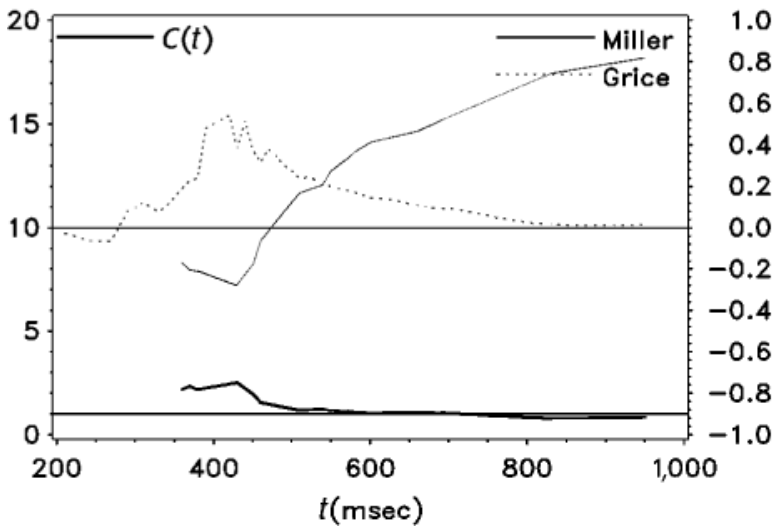

Obs. 2: Faces, Inverted

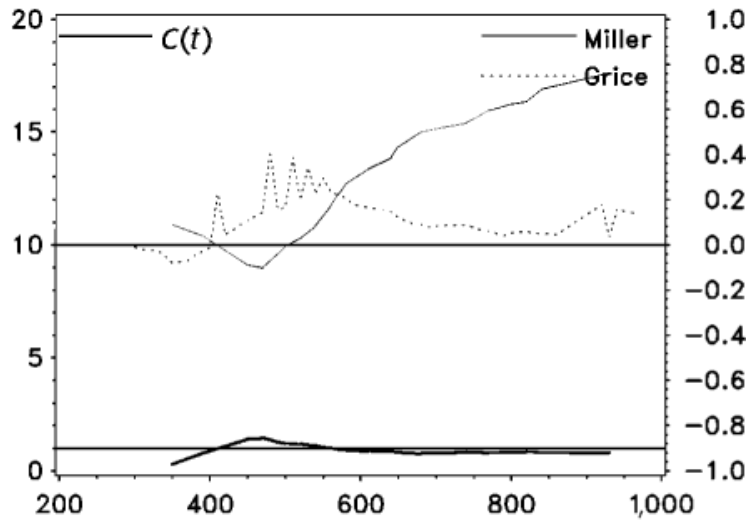

Obs. 2: Schematic, Inverted

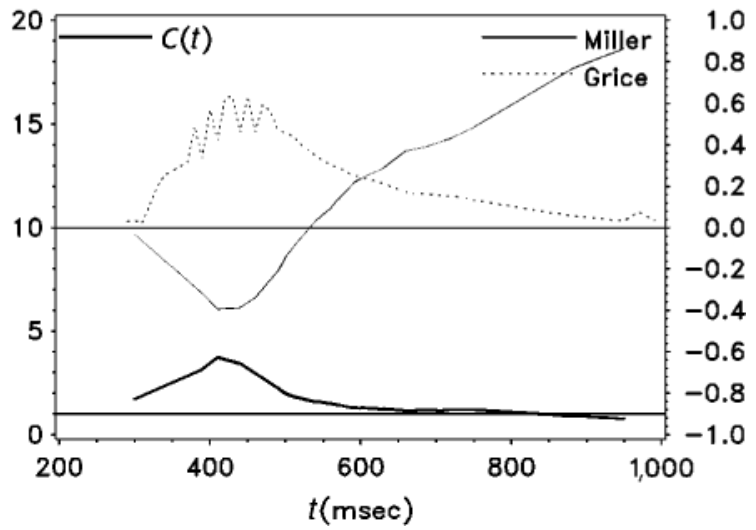

Figure 8. Observer 2: Capacity coefficients (Equation 3) and values of the Miller (Equation 4) and the Grice (Equation 5) inequalities, for the two stimulus classes in each of the two orientations.

parallel, unlimited-capacity to supercapacity processing, all as assumed by the dual-mode hypothesis. However, the data contradict the assumption of independence in rates of processing featural and configural information. The one remaining assumption critical to his hypothesis pertains to the effect of orientation on the ability to use featural and configural information in face and nonface stimuli. The dual-mode hypothesis assumes that configural information is best used in upright faces, whereas configural information is best used in inverted faces.

The data from the trials involving only one unambiguous change were examined for evidence consistent with this assumption, using a set of 2 (change type: featural or configural) $\times 2$ (orientation: upright or inverted) ANOVAs, performed separately for each type of stimulus (face or schematic) and each observer. The results expected from this analysis, on the basis of the assumptions of the dual-mode hypothesis, are an interaction of change type and orientation for the face stimuli, but not for the schematic stimuli, with the nature of the interaction being a greater cost due to inversion for the configural information in face, relative to that in schematic, stimuli. The mean RTs pertinent to this analysis are presented in
Table 7, and the results of the ANOVAs are presented in Table 8.

As can be seen in Table 8, the interaction between change type and orientation was reliable for all $4 \mathrm{ob}-$

Table 7

Mean Response Times for the Effect of Orientation on the Trials Involving Only One Type of Change, as a Function of Orientation, for Each of the 4 Observers $\left(\mathrm{O}_{1}-\mathrm{O}_{4}\right)$

\begin{tabular}{|c|c|c|c|c|c|}
\hline Change Type & Orientation & $\mathrm{O}_{1}$ & $\mathrm{O}_{2}$ & $\mathrm{O}_{3}$ & $\mathrm{O}_{4}$ \\
\hline \multicolumn{6}{|c|}{ Faces } \\
\hline Configuration & $\begin{array}{l}\text { Upright } \\
\text { Inverted } \\
U-I\end{array}$ & $\begin{array}{r}503 \\
524 \\
-21\end{array}$ & $\begin{array}{r}601 \\
630 \\
-29\end{array}$ & $\begin{array}{r}560 \\
604 \\
-44\end{array}$ & $\begin{array}{r}486 \\
485 \\
1\end{array}$ \\
\hline Feature & $\begin{array}{l}\text { Upright } \\
\text { Inverted } \\
U-I\end{array}$ & $\begin{array}{r}507 \\
481 \\
26\end{array}$ & $\begin{array}{r}694 \\
671 \\
23\end{array}$ & $\begin{array}{r}524 \\
514 \\
10\end{array}$ & $\begin{array}{r}566 \\
493 \\
73\end{array}$ \\
\hline \multicolumn{6}{|c|}{ Schematics } \\
\hline Configuration & $\begin{array}{l}\text { Upright } \\
\text { Inverted } \\
U-I\end{array}$ & $\begin{array}{r}468 \\
479 \\
-11\end{array}$ & $\begin{array}{r}542 \\
596 \\
-54\end{array}$ & $\begin{array}{r}534 \\
553 \\
-19\end{array}$ & $\begin{array}{r}419 \\
392 \\
27\end{array}$ \\
\hline Feature & $\begin{array}{l}\text { Upright } \\
\text { Inverted } \\
U-I\end{array}$ & $\begin{array}{r}508 \\
517 \\
-9\end{array}$ & $\begin{array}{r}634 \\
649 \\
-15 \\
\end{array}$ & $\begin{array}{r}528 \\
580 \\
-52\end{array}$ & $\begin{array}{r}575 \\
576 \\
-1\end{array}$ \\
\hline
\end{tabular}

Note- $-U-I$ indicates the difference in RT due to inversion. 
Table 8

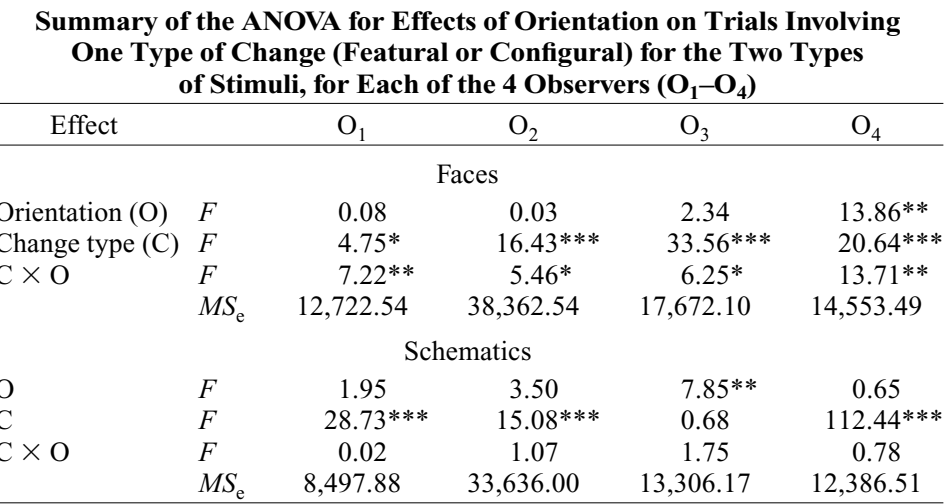

Note-Both effects and their interaction involved one degree of freedom. ${ }^{*} p<$ $.05 . \quad * * p<.01 . \quad * * * p<.001$.

servers for the face stimuli and was not reliable for any of the observers for the schematic stimuli. To better understand the nature of this interaction, Table 7 includes the value of the difference between the mean RTs for the upright and the inverted stimuli (labeled as $U-I$ ). To the extent inversion produced a decrement in the ability to process the stimulus information, this value should be negative. Considering, then, the interaction of change type and orientation for the face stimuli, it can be seen that inversion produced a decrement in performance for the configuration change for 3 of the 4 observers, while producing a benefit for the featural change. Interestingly, this same pattern was observed for the schematic stimuli. Overall, then, these results are consistent with the assumption that inversion should have more of an impact on the ability to process configural information than it should on the ability to process featural information. However, inconsistent with the assumptions of the dualmode hypothesis, this evidence is not restricted to the data for the face stimuli.

\section{DISCUSSION}

The dual-mode hypothesis assumes there are two independent sources of information in a human facefeatural and configural - that are available simultaneously in perception. The hypothesis further assumes that either source of information can contribute to the processing of a face, with the dominant source being determined (at least in great part) by the orientation of the stimulus (e.g., Bartlett \& Searcy, 1993; Searcy \& Bartlett, 1996). These assumptions correspond to informationprocessing predictions of parallel self-terminating processing demonstrating unlimited-capacity to supercapacity with a preservation of independence for the two sources. In the present study, a set of tightly related theoretical and methodological developments was used to perform a strong test of these core assumptions and is, to our knowledge, the first and only study to directly test these predictions in a way that allowed for strong-inference testing of a complete set of alternative hypotheses.

The evidence supported three of the four assumptions of the dual-mode hypothesis. That is, we found evidence for parallel self-terminating processing with unlimitedcapacity to supercapacity processing. The results falsified all forms of either serial or exhaustive processing and any form of limited-capacity processing. However, contradicting one specific assumption of the dualmode hypothesis, the data supported positive dependence in rates of processing. Of particular importance with respect to these conclusions is the consistency of the findings across observers. The inferences just noted were those supported for both facial and schematic stimuli, when presented upright or inverted, across all $4 \mathrm{ob}-$ servers. Although there were quantitative differences across observers, the qualitative inferences made on the basis of that data were remarkably consistent.

Although our results provide strong support for three of the four processing assumptions of the dual-mode hypothesis, they do call into question the extent to which the hypothesis can or should be seen as being specific to facial stimuli. As was just noted, the qualitative inferences held for both faces and schematics. In this sense, our results seem consistent with the notion that facial cognition may represent a possibly highly efficient application of general visual-processing mechanisms (as implied by, e.g., Gauthier, Tarr, Anderson, Skudlarksi, \& Gore, 1999; Tarr \& Gauthier, 2000; Wenger \& Townsend, 2001) and inconsistent with the notion of processing mechanisms that are specific to facial stimuli (e.g., Farah, 1992; Farah et al., 1998; Kanwisher, McDermott, \& Chun, 1997; Kanwisher, Stanley, \& Harris, 1999). The strength of this assertion must, of course, be tempered by the fact that our nonface stimuli intentionally possessed a structure that was quite face-like. It remains a goal for future work to determine whether objects that do not possess the types of symmetry and organization possessed by faces will show the regularities observed 
Obs. 3: Faces, Upright

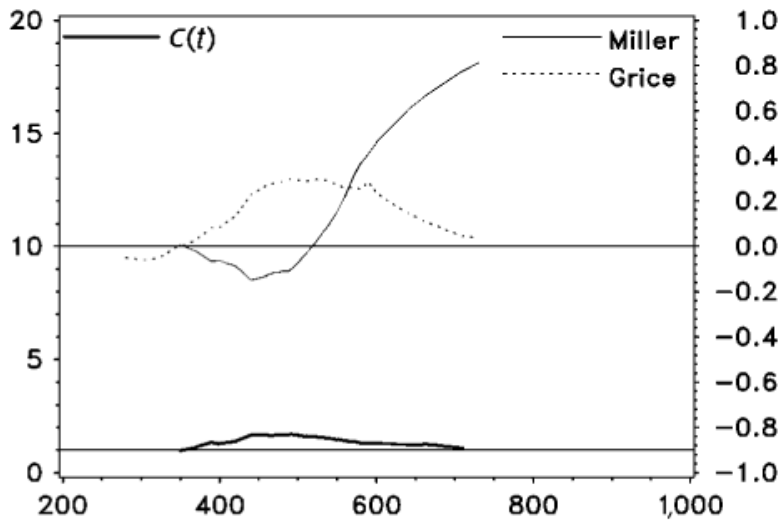

Obs. 3: Schematic, Upright

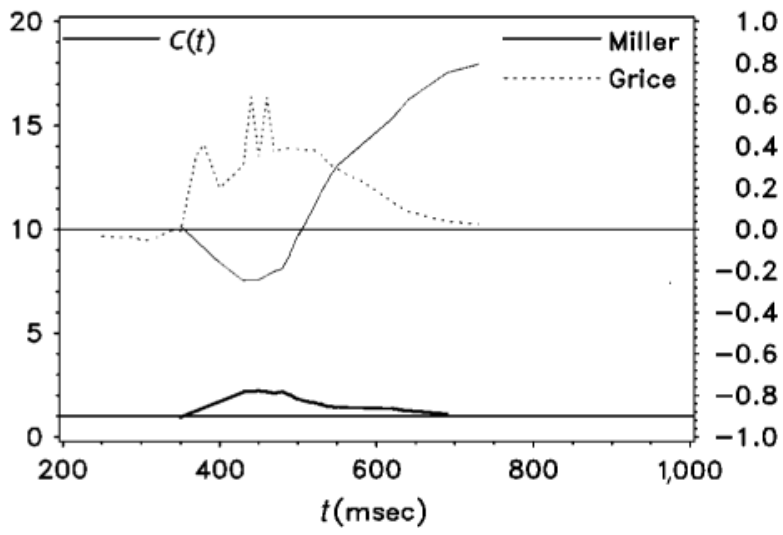

Obs. 3: Faces, Inverted

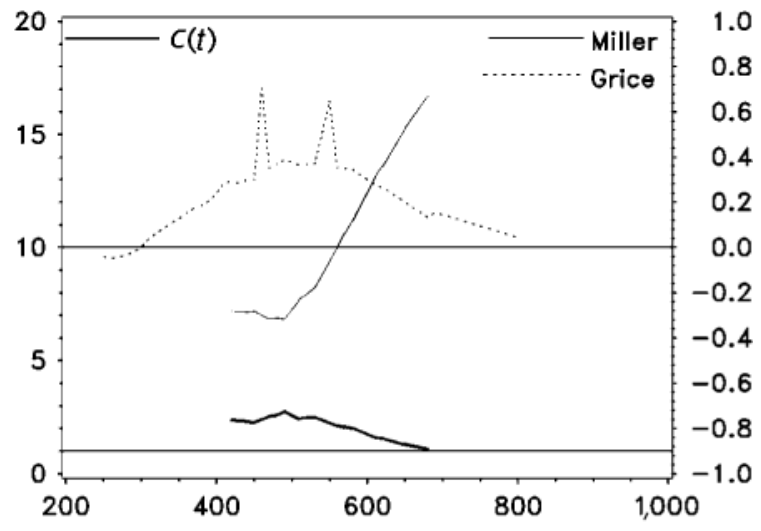

Obs.3: Schematic, Inverted

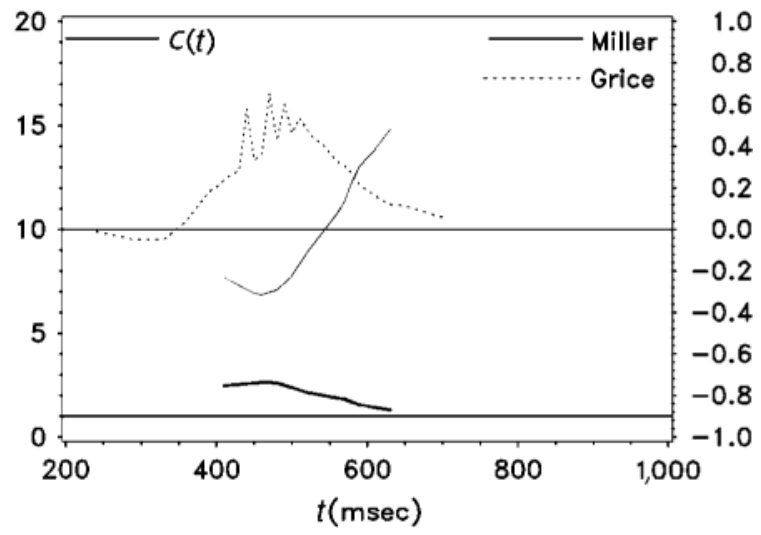

Figure 9. Observer 3: Capacity coefficients (Equation 3) and values of the Miller (Equation 4) and the Grice (Equation 5) inequalities, for the two stimulus classes in each of the two orientations.

for our schematic stimuli. However, there are strong hints (specifically, in the data of Wenger \& Townsend, 2001) suggesting that the regularities observed here will be observed in other stimuli. We should also note that such regularities, if obtained, do not argue against cortical specialization, at least at some level: it is altogether possible that two different classes of stimuli could be processed in the same way in very different cortical regions.

The effects of inversion on the ability to work with featural and configural information, observed in earlier studies and the basis of one of the core assumptions of the dual-mode hypothesis (e.g., Bartlett \& Searcy, 1993; Cabeza \& Kato, 2000; Leder \& Bruce, 1998, 2000; Valentine, 1991), were partially supported by our results. Although we did obtain the anticipated interaction of change type and orientation, specific to face stimuli, in those trials involving one type of change, we should note that we generally failed to obtain reliable main effects of inversion (see Table 8). We should caution against interpreting this as a lack of support for the dual-mode hypothesis, for at least two reasons. First, the magnitudes of the changes were selected on the basis of extensive pilot testing to equate discriminability of the featural and the configural changes. Second, the observers in our study had extensive practice with the stimuli, at a level far exceeding that used in most studies. Such extensive practice may simply have allowed our observers to become proficient with both upright and inverted stimuli. Such a possibility is bolstered by a quick examination of the data from the initial five blocks of trials (data that were not included in the results reported above), which did show reliable costs for inversion for both faces and schematics for all $4 \mathrm{ob}-$ servers. Finally, we should emphasize that the unlimitedcapacity to supercapacity results obtained for inverted faces is consistent with the assumption of the dual-mode hypothesis that both featural and configural information can aid in overcoming the negative effects of inversion.

Another issue to address with respect to the lack of a main effect due to inversion is the possible inference that our configural manipulations were not truly configural. Certainly, a number of results would suggest that a critical test for determining whether a manipulation was truly configural would be to determine whether a potentially configural change is diminished in its impact by in- 
Obs. 4: Faces, Upright

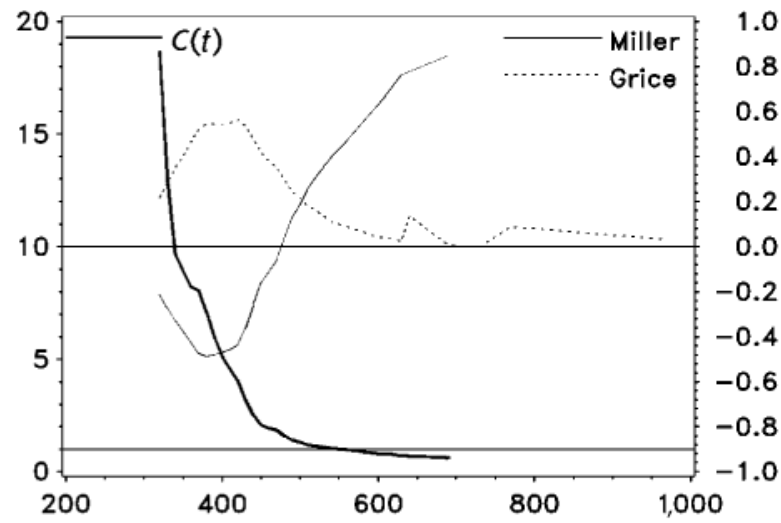

Obs. 4: Schematic, Upright

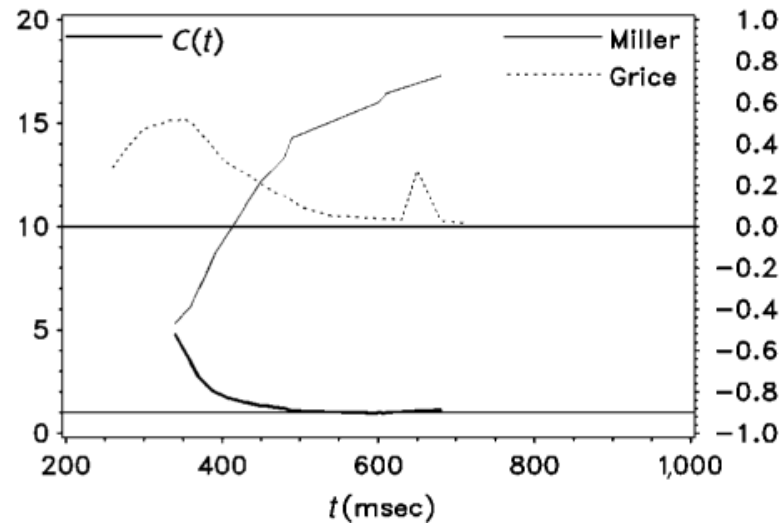

Obs. 4: Faces, Inverted

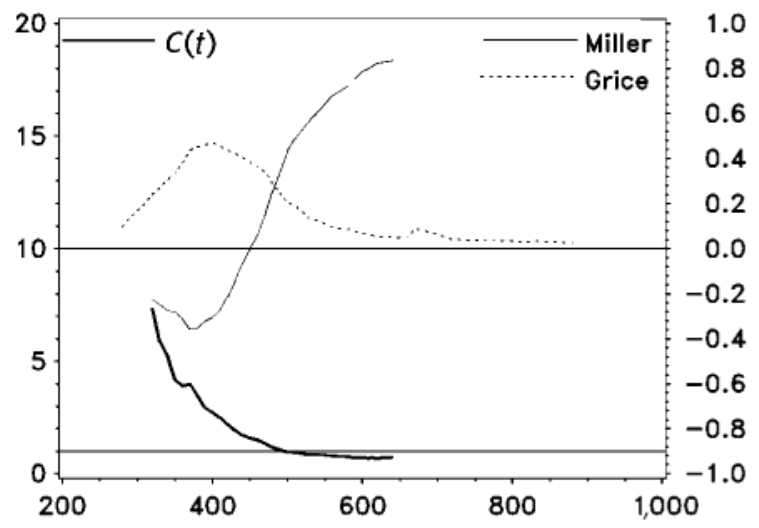

Obs. 4: Schematic, Inverted

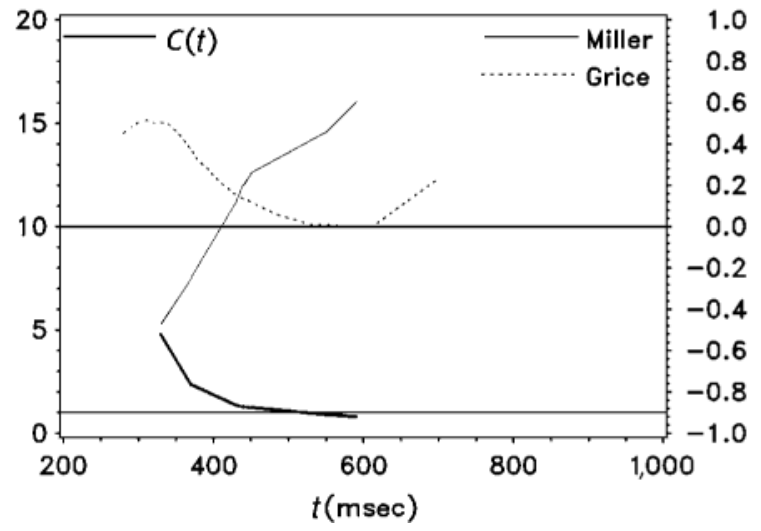

Figure 10. Observer 4: Capacity coefficients (Equation 3) and values of the Miller (Equation 4) and the Grice (Equation 5) inequalities, for the two stimulus classes in each of the two orientations.

version. If inversion does not produce a reliable decrement in the impact of the change, that change must not be a configural change. Although intuitively appealing, such an approach is unfortunately circular. To avoid this kind of circularity, we chose to follow the example of earlier studies and define our manipulation in terms of a change to the internal geometry of the face (as in, e.g., Searcy \& Bartlett, 1996). Our change was one that was both physically possible in a real face and minimally grotesque, while still being consistent with the definition of a configural change. However, we should also note that our manipulation of configuration could be interpreted as a featural change, since the overall position of the eyes was not altered. Although we would disagree with this interpretation (since it hinges in no small part on the definition of a feature), we do acknowledge the potential difference in interpretation and think that this difference may be a good point of departure for a focused debate on these and related issues.

To our knowledge, the present study is the most rigorous test of the dual-mode hypothesis to date and, therefore, provides the strongest test of and, consequently, support for three of its four core assumptions. It is also the first exploration of the hypothesis to allow for stronginference tests of a complete set of alternative hypotheses. The ability to perform these tests draws on more than 3 decades of theoretical and empirical advances (based on critical contributions from Schweickert, 1978, 1983; Schweickert et al., 2000; Schweickert \& Townsend, 1989; Townsend, 1972, 1974; Townsend \& Ashby, 1978; Townsend \& Nozawa, 1995; and Townsend \& Schweickert, 1989, among others). It is our hope that this study, like others demonstrating the application of experimental methodologies with strong links to formally specified theories of psychological information and processes (e.g., Ashby, Alfonso-Reese, Turken, \& Waldron, 1999; Maddox \& Bogdanov, 2000; Thomas, 1996; Townsend \& Nozawa, 1995; Wenger \& Ingvalson, 2002, 2003; Wenger \& Townsend, 2001), will lead to strong tests of a variety of hypotheses and, thus, enliven the ongoing empirical and theoretical discussions of facial cognition.

\section{REFERENCES}

Ashby, F. G., Alfonso-Reese, L. A., Turken, A. U., \& Waldron, E. M. (1999). A neuropsychological theory of multiple systems in category learning. Psychological Review, 105, 442-481. 
Ashby, F. G., \& Townsend, J. T. (1986). Varieties of perceptual independence. Psychological Review, 93, 154-179.

BARTLETT, J. C., \& SEARCY, J. (1993). Inversion and configuration of faces. Cognitive Psychology, 25, 281-316.

Bruce, V., \& Young, A. (1986). Understanding face recognition. British Journal of Psychology, 77, 305-327.

BuRTON, A. M., \& BRUCE, V. (1992). I recognize your face but I can't remember your name: A simple explanation? British Journal of Psychology, 83, 45-60.

BURTON, A. M., BRUCE, V., \& Johnston, R. A. (1990). Understanding face recognition with an interactive activation model. British Journal of Psychology, 81, 361-380.

CABEZA, R., \& KATO, T. (2000). Features are also important: Contributions of featural and configural processing to face recognition. Psychological Science, 11, 429-433.

CAREY, S., \& DiAMOND, R. (1977). From piecemeal to configurational representation of faces. Science, 195, 312-314.

Colonius, H., \& Townsend, J. T. (1997). Activation-state representation of models for the redundant-signals-effect. In A. A. J. Marley (Ed.), Choice, decision, and measurement: Essays in honor of R. Duncan Luce (pp. 245-254). Mahwah, NJ: Erlbaum.

Diamond, R., \& CAREY, S. (1986). Why faces are and are not special: An effect of expertise. Journal of Experimental Psychology: General, 115, 107-117.

DIEDERICH, A. (1995). Intersensory facilitation of reaction time: Evaluation of counter and diffusion coactivation models. Journal of Mathematical Psychology, 39, 197-215.

Dzhafarov, E. N. (1997). Process representations and decompositions of response times. In A. A. J. Marley (Ed.), Choice, decision, and measurement: Essays in honor of R. Duncan Luce (pp. 255-277). Mahwah, NJ: Erlbaum.

FARAH, M. J. (1992). Is an object an object an object? Cognitive and neuropsychological investigations of domain-specificity in visual object recognition. Current Directions in Psychological Science, 1, 164-169.

Farah, M. J., Wilson, K. D., Drain, M., \& Tanaka, J. N. (1998). What is "special" about face perception? Psychological Review, 105, 482498.

Faulkner, T. F., Rhodes, G., Palermo, R., Pellicano, E., \& FerguSON, D. (2002). Recognizing the un-real McCoy: Priming and the modularity of face recognition. Psychonomic Bulletin \& Review, 9 , 327-334.

Forster, K. I., \& Forster, J. C. (2003). DMDX: A Windows display program with millisecond accuracy. Behavioral Research Methods, Instruments, \& Computers, 35, 116-124.

Ganel, T., \& Goshen-GotTstein, Y. (2002). Perceptual integrality of sex and identity of faces: Further evidence for the single route hypothesis. Journal of Experimental Psychology: Human Perception \& Performance, 28, 854-867.

GARNER, W. R. (1978). Aspects of a stimulus: Features, dimensions, and configurations. In E. Rosch \& B. B. Lloyd (Eds.), Cognition and categorization (pp. 99-131). Hillsdale, NJ: Erlbaum.

Gauthier, I., Tarr, M. J., Anderson, A. W., Skudlarksi, P., \& Gore, J. C. (1999). Activation of the middle fusiform "face area" increases with expertise in recognizing novel objects. Nature Neuroscience, $\mathbf{2}$, 568-573.

Grice, G. R., Canham, L., \& Boroughs, J. M. (1984). Combination rule for redundant information in reaction time tasks with divided attention. Perception \& Psychophysics, 35, 451-463.

Grice, G. R., Canham, L., \& GWYNnE, J. W. (1984). Absence of a redundant-signals effect in a reaction time task with divided attention. Perception \& Psychophysics, 36, 565-570.

Hosie, J. A., Ellis, H. D., \& HaIG, N. D. (1988). The effect of feature displacement on the perception of well-known faces. Perception, $\mathbf{1 7}$, 461-474.

Hughes, H. C., \& TownsEnd, J. T. (1998). Varieties of binocular interaction in human vision. Psychological Science, 9, 53-60.

KADLEC, H., \& TownSEND, J. T. (1992a). Implications of marginal and conditional detection parameters for the separabilities and independence of perceptual dimensions. Journal of Mathematical Psychology, 36, 325-374.
KADLEC, H., \& TownSEND, J. T. (1992b). Signal detection analysis of dimensional interactions. In F. G. Ashby (Ed.), Multidimensional models of perception and cognition (pp. 181-228). Hillsdale, NJ: Erlbaum.

Kanwisher, N., McDermott, J., \& Chun, M. M. (1997). The fusiform face area: A module in human extrastriate cortex specialized for face perception. Journal of Neuroscience, 17, 4302-4311.

Kanwisher, N., Stanley, D., \& Harris, A. (1999). The fusiform face area is selective for faces not animals. NeuroReport, 10, 183-187.

Krantz, D. H., Luce, R. D., Suppes, P., \& Tversky, A. (1971). Foundations of Measurement: Vol. 1. Additive and polynomial representations. New York: Academic Press.

Leder, H., \& Bruce, V. (1998). Local and relational aspects of face distinctiveness. Quarterly Journal of Experimental Psychology, 51A, 449-473.

LEDER, H., \& BRUCE, V. (2000). When inverted faces are recognized: The role of configural information in face recognition. Quarterly Journal of Experimental Psychology, 53A, 513-536.

LoGAN, G. D. (1992). Shapes of reaction time distributions and shapes of learning curves: A test of the instance theory of automaticity. Journal of Experimental Psychology: Learning, Memory, \& Cognition, 18, 883-914.

LUCE, R. D. (1986). Reaction times: Their role in inferring elementary mental organization. New York: Oxford University Press.

Macho, S., \& Leder, H. (1998). Your eyes only? A test of interactive influence in the processing of facial features. Journal of Experimental Psychology: Human Perception \& Performance, 24, 1486-1500.

MADDOX, W. T., \& BogDANOV, S. V. (2000). On the reTation between decision rules and perceptual representation in multidimensional perceptual categorization. Perception \& Psychophysics, 62, 984-997.

McClelland, J. L. (1979). On the time relations of mental processes: An examination of systems of processes in cascade. Psychological Review, 86, 287-330.

MiLGRAM, P. (1997). A spectacle-mounted liquid-crystal tachistoscope. Behavior Research Methods, Instruments, \& Computers, 19, 449-456.

MiLler, J. O. (1982). Divided attention: Evidence for coactivation with redundant signals. Cognitive Psychology, 14, 247-279.

Miller, J. O. (1991). Channel interaction and the redundant-targets effect in bimodal divided attention. Journal of Experimental Psychology: Human Perception \& Performance, 17, 160-169.

MordKoff, J. T., \& EgETH, H. E. (1993). Response time and accuracy revisited: Converging support for the interactive race model. Journal of Experimental Psychology: Human Perception \& Performance, $\mathbf{1 9}$, 981-991.

MORDKOFF, J. T., \& YANTIS, S. (1991). An interactive race model of divided attention. Journal of Experimental Psychology: Human Perception \& Performance, 17, 520-538.

Parks, T. E., Coss, R. C., \& Coss, C. S. (1985). Thatcher and the cheshire cat: Context and the processing of facial features. Perception, 14, 747-754.

PLATT, J. R. (1964). Strong inference. Science, 146, 347-353.

Popper, K. R. (1935). Logik der Forschung. Berlin: Springer-Verlag.

Rhodes, G. (1988). Looking at faces: First-order and second-order features as determinates of facial appearance. Perception, 17, 43-63.

Rhodes, G., Brake, S., \& Atkinson, A. P. (1993). What's lost in inverted faces. Cognition, 47, 25-57.

SCHWEICKERT, R. (1978). A critical path generalization of the additive factor method: Analysis of a Stroop task. Journal of Mathematical Psychology, 18, 105-139.

SCHWEICKERT, R. (1983). Latent network theory: Scheduling of processes in sentence verification and the Stroop effect. Journal of Experimental Psychology: Learning, Memory, \& Cognition, 9, 353-383.

SCHWEICKERT, R., Giorgini, M., \& Dzhafarov, E. (2000). Selective influence and response time cumulative distribution functions in serial-parallel task networks. Journal of Mathematical Psychology, 44, 504-535.

SCHWEICKERT, R., \& Townsend, J. T. (1989). A trichotomy method: Interactions of factors prolonging sequential and concurrent mental processes in stochastic PERT networks. Journal of Mathematical Psychology, 33, 328-347.

SEARCY, J. H., \& BARTLETT, J. C. (1996). Inversion and processing of com- 
ponent and spatial-relational information in faces. Journal of Experimental Psychology: Human Perception \& Performance, 22, 904-915.

SERGENT, J. (1984). An investigation of component and configural processes underlying face recognition. British Journal of Psychology, 75, 221-242.

SUZUKI, S., \& CAVANAGH, P. (1995). Facial organization blocks access to low-level features: An object inferiority effect. Journal of Experimental Psychology: Human Perception \& Performance, 21, 901-913.

TANAKA, J. W., \& FARAH, M. J. (1993). Parts and wholes in face recognition. Quarterly Journal of Experimental Psychology, 46A, 225-245.

TANAKA, J. W., \& SengCo, J. A. (1997). Features and their configuration in face recognition. Memory \& Cognition, 25, 583-592.

TARr, M. J., \& GaUthier, I. (2000). Ffa: A flexible fusiform area for subordinate-level visual processing automated by expertise. Nature Neuroscience, $\mathbf{3}, 764-769$.

THOMAs, R. D. (1995). Gaussian general recognition theory and perceptual independence. Psychological Review, 102, 192-200.

Thomas, R. D. (1996). Separability and independence of dimensions within the same-different judgment task. Journal of Mathematical Psychology, 40, 318-341.

THOMPSON, P. (1980). Margaret Thatcher: A new illusion. Perception, 9, 483-484.

TowNSEND, J. T. (1972). Some results concerning the identifiability of parallel and serial processes. British Journal of Mathematical \& Statistical Psychology, 25, 168-199.

Townsend, J. T. (1974). Issues and models concerning the processing of a finite number of inputs. In B. H. Kantowitz (Ed.), Human information processing: Tutorials in performance and cognition (pp. 133168). Hillsdale, NJ: Erlbaum.

TownsEnd, J. T., \& AshBy, F. G. (1978). Methods of modeling capacity in simple processing systems. In J. Castellan \& F. Restle (Eds.), Cognitive theory (Vol. 3, pp. 200-239). Hillsdale, NJ: Erlbaum.

Townsend, J. T., \& Ashby, F. G. (1983). Stochastic modeling of elementary psychological processes. Cambridge: Cambridge University Press.

Townsend, J. T., \& Fific, M. (2004). Parallel versus serial processing and individual differences in high-speed search in human memory. Perception \& Psychophysics, 66, 953-962.

TownSEND, J. T., \& FIKES, T. (1995). A beginning quantitative taxonomy of cognitive activation systems and application to continuous flow processes (Tech. Rep. 131). Bloomington: Indiana University, Cognitive Science Program.

Townsend, J. T., \& Nozawa, G. (1995). On the spatio-temporal properties of elementary perception: An investigation of parallel, serial, and coactive theories. Journal of Mathematical Psychology, 39, 321-359.

Townsend, J. T., \& SchweICKERT, R. (1989). Toward the trichotomy method: Laying the foundation of stochastic mental networks. Journal of Mathematical Psychology, 33, 309-327.

Townsend, J. T., \& Wenger, M. J. (2004a). The serial-parallel dilemma: A case study in a linkage of theory and method. Psychonomic Bulletin \& Review, 11, 391-418.

TownSEND, J. T., \& WENGER, M. J. (2004b). A theory of interactive parallel processing: New capacity measures and predictions for a response time inequality series. Psychological Review, 111, 1003-1035.

VAlEntine, T. (1991). A unified account of the effects of distinctiveness, inversion, and race in face recognition. Quarterly Journal of Experimental Psychology, 43A, 161-204.

Webster, M. A., \& MacLin, O. H. (1999). Figural aftereffects in the perception of faces. Psychonomic Bulletin \& Review, 6, 647-653.

WeIBULL, W. (1951). A statistical distribution function of wide applicability. Journal of Applied Mechanics, 18, 293-297.

Wenger, M. J., Hill, A. M., \& Klatt, S. R. (2001, March). Tachistoscopic displays with visual occlusion spectacles: An off-the-shelf system. Paper presented at the Hoosier Mental Life Meeting, Bloomington, IN.

Wenger, M. J., \& IngValson, E. M. (2002). A decisional component of holistic encoding. Journal of Experimental Psychology: Learning, Memory, \& Cognition, 28, 872-892.

WENGER, M. J., \& INGVALSON, E. M. (2003). Preserving informational separability and violating decisional separability in facial perception and recognition. Journal of Experimental Psychology: Learning, Memory, \& Cognition, 29, 1106-1118.

Wenger, M. J., Schuster, C., Petersen, L. M., \& Petersen, R. C. (in press). Application of proportional hazards models to reaction time data. In C. Bergeman \& S. Boker (Eds.), Notre Dame series on quantitative methodologies. Mahwah, NJ: Erlbaum.

Wenger, M. J., \& Townsend, J. T. (2000). Basic response time tools for studying general processing capacity in attention, perception, and cognition. Journal of General Psychology, 127, 67-99.

Wenger, M. J., \& TownSEND, J. T. (2001). Faces as gestalt stimuli: Process characteristics. In M. J. Wenger \& J. T. Townsend (Eds.), Computational, geometric, and process perspectives on facial cognition (pp. 229-284). Mahwah, NJ: Erlbaum.

YIN, R. K. (1969). Looking at upside-down faces. Journal of Experimental Psychology, 81, 141-145.

\section{NOTES}

1.The dual-mode hypothesis is distinct and addresses very different issues from another hypothesis regarding the processing of faces: the dual route hypothesis (e.g., Bruce \& Young, 1986; Burton \& Bruce, 1992; Burton, Bruce, \& Johnston, 1990; Ganel \& Goshen-Gottstein, 2002).

2. Note that this first assumption itself requires the assumption of two sources of information. Since the work we will be reviewing, and the experimental work we will be presenting, all involve, in one way or another, manipulations of these two types of information, we take the requisite assumption of featural and configural information as a given.

3. We use featural and configural information in this discussion for obvious reasons; note, however, that the distinctions we consider can be generalized to any two (or more) sources of psychological information.

4. Note that independence in rates of processing is an issue that is logically distinct from independence (or its violation) in the final products of information processing. This second issue is addressed in characterizations of the psychological information space for a task (as in Ashby \& Townsend, 1986; Kadlec \& Townsend, 1992a, 1992b; Thomas, $1995,1996)$ and is an issue we have begun to address in other studies (Wenger \& Ingvalson, 2002, 2003).

5. Note that we are not considering such alternatives as continuous flow or cascaded systems (as in, e.g., Colonius \& Townsend, 1997; McClelland, 1979; Townsend \& Fikes, 1995). However, we should also note that in many cases, when such systems are analyzed using the measures we apply here, the results are consistent with those for more traditional (e.g., stage-based) systems (see, e.g., Townsend \& Wenger, 2004b).

6. Readers may be more familiar with the cumulative distribution function of the RT distribution, $F(t)$, than with the survivor function, $S(t)$. The two functions are simply complements of one another - that is, $S(t)=1-F(t)$ and $F(t)=1-S(t)$. Thus, although the interpretations of the two functions are different, with $S(t)$ giving the probability that a response has not yet occurred at time $t$ and $F(t)$ giving the probability that a response has occurred at or before time $t$, there is a one-to-one mapping between the two functions.

7. It is worth emphasizing that the derivations presented by Townsend and Nozawa (1995) explicitly take into consideration the effects of what are sometimes referred to as base times (e.g., motor response components of the total task time), an aspect of performance that is generally not given explicit consideration. Thus, the predictions summarized here can be interpreted as holding for observed distributions that include a base time distribution with nonzero variance.

8. It should be emphasized that the capacity coefficient (Equation 3 ) is not in any way identical to or redundant with the Miller and the Grice inequalities. Specifically, violation of the Miller inequality indicates an extreme level of supercapacity, whereas a violation of the Grice inequality indicates an extreme limitation in capacity. We provide additional detail and an example pertinent to the inference of supercapacity processing in the appendix. Townsend and Nozawa (1995) detailed the relationships between the capacity coefficient and the Miller inequality in their Proposition 6 (also Theorem 6), whereas Townsend and Wenger (2004b) consider the capacity coefficient with respect to both the Miller and the Grice inequalities in their Proposition 1 (also Theorem 1). Finally, we should 
note that the estimation and interpretation of the capacity coefficient and the Miller and the Grice inequalities assume that the influence of base times is negligible.

9. In fact, earlier work (Wenger \& Ingvalson, 2002, 2003) indicated that face-like schematics were treated the same way by observers as were other human and nonhuman faces in initial perception and later recognition.
10. Additional details are available on the World-Wide Web, at http:// www.nd.edu/ mwenger1/lab/preprints/hml2001.goggles.html.

11. More precisely, the presence of main effects would indicate that the manipulation was effective (per Krantz, Luce, Suppes, \& Tversky, 1971).

12. For pragmatic purposes, the Kolmogorov-Smirnov tests were conducted at the level of the cumulative distribution function, the complement of the survivor function - that is, $F(t)=1-S(t)$.

\section{APPENDIX}

\section{Base Times and Potential Bias in Estimating $\boldsymbol{C}(\boldsymbol{t})$}

A reviewer of an earlier version of this article rightly noted that, under certain assumptions, the estimated value of $C(t)$ will be systematically lower than the true value. We consider two of those sets of assumptions in this section of the appendix. The first set of assumptions concerns the capacity of the base time processes themselves, and the second concerns the variance of the base time processes, relative to the component processes.

Consider, first, the assumptions regarding total base times. One possible assumption would be that total base times are independent of total number of inputs. Although appealing, this assumption is essentially one of unlimited capacity for the base times, meaning that, if one were to adopt this assumption, one would need to assume unlimited capacity for base time processes in order to estimate capacity for other (central) processes. An alternative assumption would be that the total base time contribution is a function of the total number of inputs.

To examine the contrasting effects of these two different assumptions on the estimate of $C(t)$, we performed a simple numerical simulation. We assumed a system that worked with either one or two inputs. We modeled the processing times for each of the inputs, using a two-parameter Weibull (1951) distribution:

$$
f(t)=\frac{\gamma t^{\gamma-1}}{\lambda^{\gamma}} \exp \left[-\left(\frac{t}{\lambda}\right)^{\gamma}\right],
$$

where $\lambda$ is a location parameter and $\gamma$ is a shape parameter. For this simulation, we set $\lambda=45$ and $\gamma=1.9$, which yields a long-tailed distribution typical of RT distributions (see Logan, 1992; Luce, 1986; Wenger, Schuster, Petersen, \& Petersen, in press). We modeled base times using a normal (Gaussian) distribution, with $\mu=150$ and $\sigma=25$. Observed RTs for the single-target trials were simply the processing time plus the base time. Observed RTs for the double-target trials were the minimum of the two process times (i.e., an independent parallel self-terminating process, or horse race, corresponding to an OR response rule), with either one or two base time contributions. We generated 10,000 simulated trials for each of the single-and the doubletarget conditions. Independent of base times, the expected value for $(C t)$ for this system should be 1.00.

The top panel of Figure A1 displays the results for this simulation. As can be seen, the assumption of unlimited capacity for base times leads to a conservative bias for assumptions regarding central processes; that is, if one assumes unlimited capacity for the base time processes, the likelihood of incorrectly inferring limited capacity for central processes is increased. Alternatively, if one assumes that the base time contribution is a function of the number of inputs, the bias in the estimate of $C(t)$ disappears. We should note that we are not arguing in favor of either of these assumptions. In fact, neither set of assumption may be psychologically plausible. Rather, we want to emphasize how different assumptions regarding the relationship between base times and the component times can drastically change the resulting estimates.

The variance of the base times can also have a profound impact on the bias present in the estimate of $C(t)$. To illustrate this, we performed a second simulation, in which we allowed the standard deviation of the base time distribution to take on one of four values: $75,150,225$, or 300 . We assumed that the component processes were distributed according to the two-parameter Weibull (Equation 6) and that the double-target trials reflected a single base time contribution (i.e., we assumed unlimited capacity for the base time processes). We generated a total of 10,000 observations for the single- and double-target trials. Again, independent of base times, the expected value for $(C t)$ for this system should be 1.00. The bottom panel of Figure A1 displays the results for this second simulation. As the variance for the base time distribution increases, the conservative bias in the estimate of $C(t)$ increases, at least when one assumes unlimited capacity for the base time processes.

\section{Relating $\boldsymbol{C}(t)$ and the Miller Inequality}

As was noted in the text, there is a systematic relationship between the Miller inequality (Equation 4) and the capacity coefficient $C(t)$ (Equation 3 ). The relationship between these two measures is important to consider when the data suggest supercapacity processing, as is the case in the present study. However, these two measures are not identical. Townsend and Nozawa (1995) presented a formal analysis of the relationship be- 

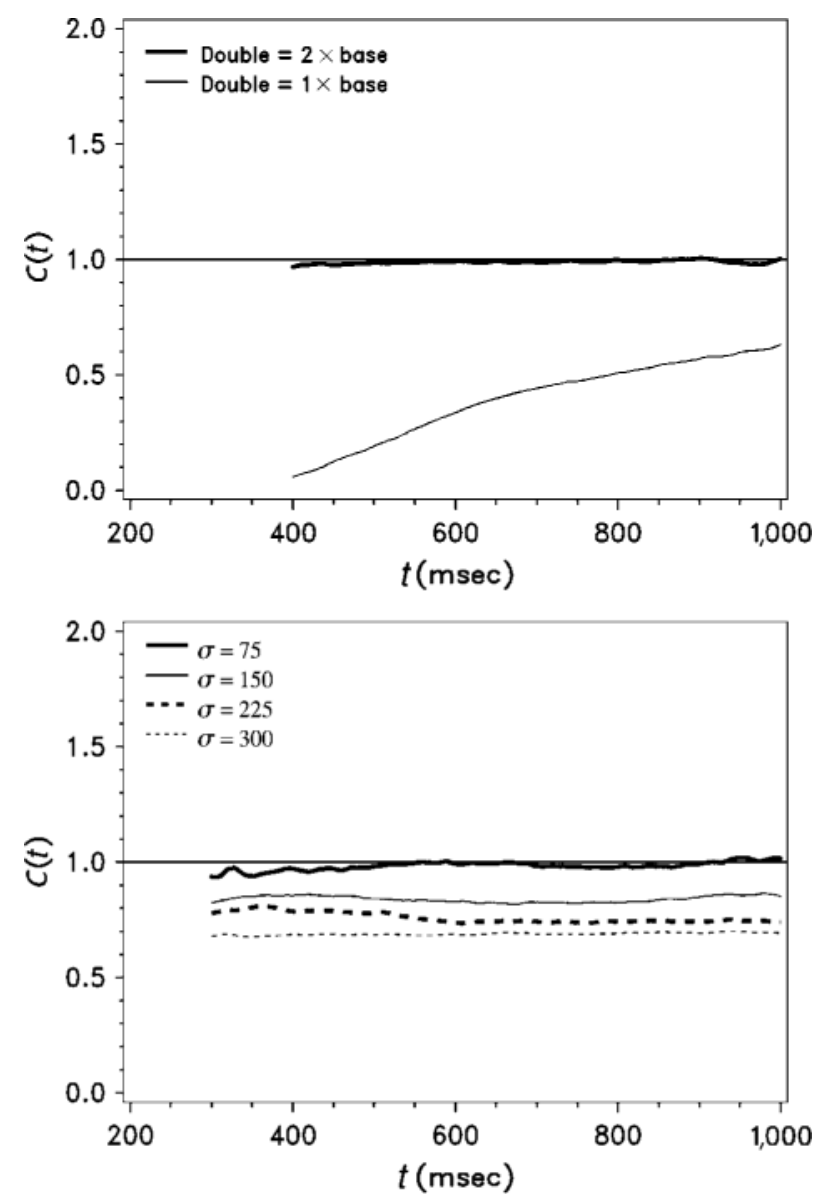

Figure A1. Top: comparison of the value of $C(t)$ under the assumptions of either one (double $=1 \times$ base) or two (double $=2 \times$ base) base time contributions to the double-target trials. Bottom: effect of changing the standard deviation $(\sigma)$ of the base time process on the estimated value of $C(t)$.

tween $C(t)$ and the Miller inequality (see also Townsend \& Wenger, 2004b). Their analysis can be summarized in the following two points. (1) If Miller's inequality is violated at some time $t$, the system is supercapacity - that is, $C_{\mathrm{o}}(t)>1$ at that time $t$. (2) If the system is supercapacity for an interval of time near $t=0$, there is a window of time within that interval where Miller's inequality is violated. That is, if one knows that Miller's inequality is violated at some time $t$, one knows that $C(t)>1$ for that time $t$. Alternatively, if one knows that $C(t)>1$ for some window of time near $t=0$, one can also infer that Miller's inequality is violated somewhere in that same time interval. Consequently, a violation of the Miller inequality is best interpreted as suggesting extreme supercapacity processing, and thus the two measures can be seen as providing converging, not identical, sources of evidence for inferences regarding capacity.

To illustrate this, we simulated two versions of a self-terminating parallel processing system using the simulation methods described in Townsend and Wenger (2004b). The first system corresponded to a standard independent, parallel, self-terminating system and, so, should evidence unlimited-capacity processing [i.e., $C(t)=1$ for the range of obtained RTs]. The second system incorporated a positive dependency between the processing channels; as a consequence, this system can be thought of as being "designed" to evidence super capacity [i.e., $C(t)>1$ ] processing (see Townsend \& Wenger, 2004b). The values of $C(t)$ and the Miller inequality for these two systems are presented in Figure A2. 


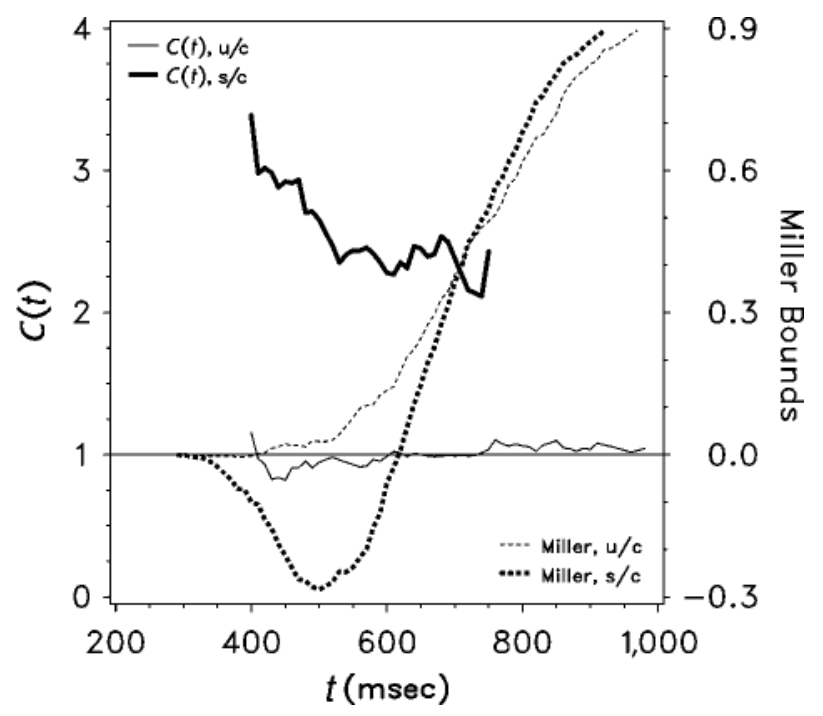

Figure A2. Comparison of $C(t)$ (Equation 3) and the Miller inequality (Equation 4) for two simulated systems: an unlimitedcapacity (u/c), independent, parallel self-terminating system and a supercapacity (s/c) parallel self-terminating system.

The two thin lines present the results for the unlimited-capacity system. As can be seen in the figure, the value of $C(t)$ for this system (thin solid line) hovers around 1.00 for the entire range of simulated RTs, and the value of the Miller inequality (thin dashed line) is greater than 0 (indicating no violation) for the corresponding range. The two thick lines present the results for the supercapacity system. As was expected, the value of $C(t)$ (thick solid line) for this system is well above 1.00 for the range of simulated RTs. Miller's inequality (thick dashed line) is less than 0 for a portion of the range, indicating a violation, and is greater than or equal to 0 (indicating no violation) for the longest simulated RTs. Interested readers should consult pp. 354-355 of Townsend and Nozawa (1995) for the formal analysis and additional details.

(Manuscript received January 11, 2004;

revision accepted for publication February 15, 2004.) 